\title{
A generalized family of Post-Newtonian Dedekind Ellipsoids
}

\author{
Norman Gürlebeck \\ ZARM, University of Bremen, Germany, EU \\ Institute of Theoretical Physics, Charles University, Prague, Czech Republic, EU \\ norman.guerlebeck@zarm. uni-bremen.de \\ David Petroff \\ Coordination Centre for Clinical Trials, University of Leipzig, Leipzig, Germany, EU \\ david.petroff@zks. uni-leipzig.de
}

\begin{abstract}
We derive a family of post-Newtonian (PN) Dedekind ellipsoids to first order. They describe non-axially symmetric, homogeneous, and rotating figures of equilibrium. The sequence of the Newtonian Dedekind ellipsoids allows for an axially symmetric limit in which a uniformly rotating Maclaurin spheroid is recovered. However, the approach taken by Chandrasekhar \& Elbert (1974) to find the PN Dedekind ellipsoids excludes such a limit. In Gürlebeck \& Petroff (2010), we considered an extension to their work that permits a limit of 1 PN Maclaurin ellipsoids. Here we further detail the sequence and demonstrate that a choice of parameters exists with which the singularity formerly found in Chandrasekhar \& Elbert (1974) along the sequence of PN Dedekind ellipsoids is removed.
\end{abstract}

Subject headings: Non-axisymmetric figures of equilibrium, Post-Newtonian approximation, Dedekind ellipsoids

\section{Introduction}

The modeling of equilibrium figures is one major goal in astrophysics. In the case of compact objects, relativistic effects become relevant and it is necessary to treat them using General Relativity. Exact solutions in this field are rare, and one is generally required to resort to approximation or numerical methods. A detailed treatment of these issues can be found in Meinel et al. (2008), Friedman \& Stergioulas (2013), where the latter includes important discussions of stability. Although it is the numerical approach, with its many possibilities for taking into account the properties of matter, that provides the most accurate means of modeling real astrophysical objects, it is essential that one pursues analytic methods in order to gain deeper insight into the nature of the theory of relativity and how it differs from Newtonian theory. One of the most fruitful avenues of research has always been the treatment of homogeneous matter, where it is precisely the extreme simplification that has permitted great headway. Here we consider one such object, the post-Newtonian (PN) Dedekind ellipsoid, in some depth, since we believe that doing so provides new insights by correcting previous errors and by setting out along a path that may provide new answers concerning the nature of stationary solutions within relativity.

In Newtonian gravity, closed form solutions are known for various ellipsoidal figures of equilibrium including the Maclaurin spheroids (rigidly rotating, axially symmetric, and stationary), the tri-axial Jacobi ellipsoids (rigidly rotating, nonaxially symmetric, and time independent in a rigidly rotating frame), the tri-axial Dedekind ellipsoids (non-axially symmetric and stationary), and the tri-axial Riemann ellipsoids (in general: non-axially symmetric and time independent in a 
rigidly rotating frame). For a summary of their properties see Hagihara (1970); Chandrasekhar (1987). The fluids described by these solutions are homogeneous and have a velocity field, which is linear in Cartesian coordinates. Moreover, they allow for the ratio of two half axes of the ellipsoid to be adjusted arbitrarily giving rise to one parameter families of solutions. For tri-axial ellipsoids, the third semi-axis is uniquely determined by choosing this ratio. The Jacobi sequence and the Dedekind sequence branch off from the Maclaurin sequence thereby allowing an axially symmetric and rigidly rotating limit.

In the search for analogous figures of equilibrium in General Relativity, there is still much work to do. However, for all of the aforementioned Newtonian families of figures of equilibrium, a post-Newtonian (PN) approximation was found in the sequence of papers Chandrasekhar (1965a, 1967a, b, 1970, 1971b a); Chandrasekhar \& Elbert (1974). Hereafter we call these articles Papers I and, if we refer only to the last one, Paper II. The Maclaurin sequence was also studied in Bardeen (1971); Petroff (2003b). In the latter paper, an algorithm was given that allows one to obtain the PN approximation to the Maclaurin ellipsoids to arbitrary order. Since the Newtonian Jacobi ellipsoid has a time dependent quadrupole moment, the energy loss due to gravitational wave emission can be estimated. This was done in Chandrasekhar (1970). Thus, they cannot describe figures of equilibrium in General Relativity, assuming that the Newtonian limit exists. In fact, it was shown that the non-radiating final state is the Maclaurin ellipsoid at the bifurcation point assuming that the Jacobi ellipsoids evolve along the Jacobi sequence. Similarly, the Riemannian ellipsoids also lose energy due to gravitational wave emission. The irrotational Riemann ellipsoids were investigated to $1 \mathrm{PN}$ order in Taniguchi et al. (1998). However, the Newtonian Dedekind ellipsoids are stationary. Although they are non-axially symmetric they keep their form due to internal motion - each fluid element moves along an ellipse in a plane perpendicular to the total angular momentum of the configuration. Thus, they are a good starting point to investigate the question if stationary but not static relativistic stars are necessarily axially symmetric, cf. Lindblom (1992). If dissipative effects are not neglected, it was shown in Lindblom (1976) that this is always true, but in case of perfect fluids it is still an open question.

Before we start deriving PN Dedekind ellipsoids, we should state which properties should be satisfied by such a sequence. Obviously, they should yield the Newtonian Dedekind ellipsoids in an appropriate limit, which is guaranteed if they are used as starting point of a $\mathrm{PN}$ approximation. Moreover, the PN Dedekind ellipsoids 1 should generalize as many properties of the Newtonian Dedekind ellipsoids as possible. This includes the reflection symmetry with respect to the coordinate planes and they should approach the $1 \mathrm{PN}$ Maclaurin spheroids close to the bifurcation point. The first property is respected in Paper II but not the second. In Gürlebeck \& Petroff (2010), we showed that both requirements can be satisfied with a generalization of the ansatz for the 1 PN Dedekind ellipsoids of Paper II. In the present paper, we study the entire sequence of these figures of equilibrium and discuss their properties.

All 1 PN sequences of figures of equilibrium studied in Papers I admit singularities at certain axis ratios. This implies that in a neighborhood of these points, the $1 \mathrm{PN}$ approximation is not applicable any longer. Interestingly, these singularities along the PN Maclaurin and PN Jacobi sequences appear at axis ratios, where the Newtonian sequence has special properties. In Chandrasekhar (1967a), it was shown that the singularity along the 1 PN Maclaurin sequence coincides with the first bifurcation point along the Newtonian Maclaurin sequence of a sequence of axially symmetric, stationary, rigidly rotating and homogeneous figures of equilibrium. A conjecture stating that all the bifurcation points of such sequences are reflected by a singularity in the PN approximation, cf. Bardeen (1971), was proven in Petroff (2003b). There it was pointed out that, if the bifurcation points are ordered appropriately, then there appears a singularity in the $n$th PN order for the $n$th bifurcation point.

For the PN Jacobi sequence, see Chandrasekhar (1967b), the singularity at the $1 \mathrm{PN}$ order is re-

\footnotetext{
${ }^{1}$ Of course, the shape of the $1 \mathrm{PN}$ configuration is not necessarily that of an ellipsoid in the coordinate system chosen. However, since the corrections are small we still refer to the solution as an "ellipsoid".
} 
lated to the onset of a fourth-harmonic neutral mode of deformation of the Newtonian Jacobi ellipsoids; also here the singularities in the PN approximation of Jacobi ellipsoids is intimately tied to physical properties of the Newtonian sequence. However, the axis ratio, where the singularity obtained for the 1 PN Dedekind ellipsoids appears in Paper II, could not be identified with a special point along the Newtonian sequence by the authors of that paper. With our generalization of the ansatz for the Dedekind ellipsoids, see Gürlebeck \& Petroff (2010), we are able to show that this singularity can be removed completely, suggesting that it is only due to an ansatz, which is too restrictive. Surprisingly, Chandrasekhar \& Elbert already considered such a generalization in Footnote 2 of Paper II, but discarded it because it was not helpful in curing the singularity in their opinion.

The paper is organized as follows. We will first discuss the Newtonian solution. On the one hand, this is done to fix our notation, but will also enable us to motivate certain limits and properties for the 1 PN generalized Dedekind ellipsoids. We will also determine the exterior solution in an explicit form using ellipsoidal harmonics. In Section 3, we give the field equations for a $1 \mathrm{PN}$ self-gravitating perfect fluid solution. These are solved for the Newtonian Dedekind ellipsoids as starting point. Subsequently, we discuss the properties of this 1 parameter family in detail, in particular the singularities in parameter space. Some explicit formulae, lengthy calculations, and figures are moved to the Appendices for readability.

\section{The Newtonian solution}

The Newtonian Dedekind ellipsoids describe rotating and tri-axial ellipsoids with a homogeneous mass density. They are stationary in an inertial frame and are solutions to the coupled Poisson and Euler equations:

$$
\Delta U=-4 \pi G, \quad \nabla p=\mu(\nabla U-(\mathbf{v} \cdot \nabla) \mathbf{v}),
$$

where $U$ is the Newtonian gravitational potential, $\mu$ the homogeneous mass density (i.e., a constant) and $\mathbf{v}$ the Newtonian velocity of the particles. The surface of vanishing pressure, i.e., the surface of the configuration, is that of a tri-axial ellipsoid:

$$
S\left(x^{1}, x^{2}, x^{3}\right)=1-\sum_{i=1}^{3}\left(\frac{x^{i}}{a_{i}}\right)^{2}=0 .
$$

We have chosen $a_{1} \geq a_{2} \geq a_{3}$ without loss of generality. The three axes $a_{i}$ have to satisfy the relation

$$
a_{1}^{2} a_{2}^{2} A_{12}=a_{3}^{2} A_{3} .
$$

The index symbols $A_{i_{1} \ldots}$ and $B_{i_{1} \ldots}, i_{n} \in\{1,2,3\}$, see Chandrasekhar (1987), are defined by

$$
\begin{aligned}
z_{i} & =u+a_{i}^{2}, \quad H(u)=\frac{a_{1} a_{2} a_{3}}{\sqrt{z_{1} z_{2} z_{3}}}, \\
A_{i_{1} \ldots i_{k}} & =\int_{0}^{\infty} H(u)\left(z_{i_{1}} \cdots z_{i_{k}}\right)^{-1} d u, \\
B_{i_{1} \ldots i_{k}} & =\int_{0}^{\infty} H(u) u\left(z_{i_{1}} \cdots z_{i_{k}}\right)^{-1} d u .
\end{aligned}
$$

It is also convenient to define

$$
A_{\emptyset}=\int_{0}^{\infty} H(u) d u .
$$

These index symbols satisfy various identities that allow one to express any $A_{i_{1} \ldots}$ and $B_{i_{1} \ldots}$ as a linear combination of $A_{1}$ and $A_{2}$, see Chandrasekhar (1987). We introduce the dimensionless axis ratios $\bar{a}_{2}=\frac{a_{2}}{a_{1}}$ and $\bar{a}_{3}=\frac{a_{2}}{a_{1}}$. The index symbols are homogeneous functions in $a_{1}$ :

$$
\begin{aligned}
& A_{i_{1} \ldots i_{k}}\left(a_{1}, a_{2}, a_{3}\right)=a_{1}^{2-2 k} A_{i_{1} \ldots i_{k}}\left(1, \bar{a}_{2}, \bar{a}_{3}\right) \\
& B_{i_{1} \ldots i_{k}}\left(a_{1}, a_{2}, a_{3}\right)=a_{1}^{4-2 k} B_{i_{1} \ldots i_{k}}\left(1, \bar{a}_{2}, \bar{a}_{3}\right) .
\end{aligned}
$$

The dimensionless index symbols $A_{i_{1} \ldots i_{k}}\left(1, \bar{a}_{2}, \bar{a}_{3}\right)$ and $B_{i_{1} \ldots i_{k}}\left(1, \bar{a}_{2}, \bar{a}_{3}\right)$ will be denoted by $\bar{A}_{i_{1} \ldots i_{k}}$ and $\bar{B}_{i_{1} \ldots i_{k}}$, respectively. Thus, Equation (3) in dimensionless form is given by $\bar{a}_{2}^{2} \bar{A}_{12}=\bar{a}_{3}^{2} \bar{A}_{3}$. It can be solved numerically and determines $\bar{a}_{3}$ as a function of $\bar{a}_{2}$ independently of $a_{1}$ (see Chandrasekhar (1987) for a table of values for $\bar{a}_{3}$ given $\bar{a}_{2}$ ). Hence, the free parameters of this solution are $\bar{a}_{2} \in[0,1]$, $a_{1}$ and the mass density $\mu$ or, alternatively, the total mass of the configuration $M=\frac{4}{3} \pi \mu a_{1} a_{2} a_{3}$. We further introduce a dimensionless constant $\bar{\Omega}$ and a constant $\Omega$ via

$$
\bar{\Omega}=\sqrt{2 \bar{B}_{12}}=\frac{\Omega}{\sqrt{\pi G \mu}} .
$$


The solution of Equations (1) with a surface given by Equations (2) and (3) reads in the interior, cf. Chandrasekhar (1987),

$$
\begin{aligned}
& \mathbf{v}=\sqrt{\pi G \mu} \bar{\Omega}\left(-\frac{x^{2}}{\bar{a}_{2}}, \bar{a}_{2} x^{1}, 0\right), \\
& U=2 \pi G \mu\left(a_{1}^{2} \bar{A}_{\emptyset}-\sum_{i=1}^{3} \bar{A}_{i}\left(x^{i}\right)^{2}\right), \\
& p=\pi G \mu^{2} a_{3}^{2} \bar{A}_{3}\left(1-\sum_{i=1}^{3}\left(\frac{x^{i}}{a_{i}}\right)^{2}\right) .
\end{aligned}
$$

\subsection{Limiting cases of the Dedekind se- quence}

Several limits are possible in the parameter space of this family of solutions. Focusing on the axis ratio $\bar{a}_{2}$, there are the limits $\bar{a}_{2} \rightarrow 1$ and $\bar{a}_{2} \rightarrow$ 0 . In the latter case, we have $\bar{a}_{3} \rightarrow 0$, too. In the first case, the Dedekind ellipsoid approaches the Maclaurin ellipsoid with $\bar{a}_{3}^{M}=0.5827 \ldots$ marking the well-known bifurcation point along the Maclaurin sequence. At this point, both the Jacobi and the Dedekind ellipsoids branch off. In this limit, the free parameters $a_{1}$ and the mass density $\mu$ can be prescribed as an arbitrary function of $\bar{a}_{2}$ leading to several qualitatively different possibilities depending on the behavior of these functions. For instance, if $a_{1} \rightarrow 0$, which implies $a_{2} \rightarrow 0$ as well as $a_{3} \rightarrow 0$, and if moreover $M \rightarrow M_{0}<\infty$, then the Maclaurin ellipsoids contract to a point particle with mass $M_{0}$. In this limit, the velocity fields vanish as well. If on the other hand, $a_{1} \rightarrow \infty$, which implies $a_{2}, a_{3} \rightarrow \infty$, and $\mu$ approaches some value $0<\mu_{0}<\infty$ the entire space is filled with a rigidly rotating perfect fluid. Of course, this solution becomes unphysical for radii, where the fluid elements have an orbital velocity greater than the velocity of light. In the limit, where both, $a_{1}$ and $\mu$, approach some finite and positive values $a_{1,0}$ and $\mu_{0}$, a Maclaurin ellipsoid is obtained. Here as well, $a_{1,0}$ is restricted by the physical requirement of subluminal motion.

Let us turn our attention to $\bar{a}_{2} \rightarrow 0$. In order to discuss this limit, we shall need to use the following expansions 2

$$
\begin{aligned}
\bar{a}_{2}= & \bar{a}_{3}+\bar{a}_{3}^{3}\left(\ln 4-3-2 \ln \bar{a}_{3}\right)+o\left(\bar{a}_{3}^{4}\right), \\
\bar{A}_{1}= & -2 \bar{a}_{3}^{2}\left(\ln \frac{\bar{a}_{3}}{2}+1\right)+\bar{a}_{3}^{4}\left(\operatorname { l n } \overline { a } _ { 3 } \left(4 \ln \bar{a}_{3}+\right.\right. \\
& \left.9-8 \ln 2)+\frac{13}{2}+\ln 2(\ln 16-9)\right)+o\left(\bar{a}_{3}^{5}\right), \\
\bar{A}_{2}= & 1+\bar{a}_{3}^{2}\left(2 \ln \bar{a}_{3}+\frac{5}{2}-\ln 4\right)+o\left(\bar{a}_{3}^{3}\right) .
\end{aligned}
$$

The expansions of the other index symbols can be inferred from the recursion relations given in Chandrasekhar (1987), cf. the comment after Equation (4).

In the most interesting case, where $a_{1} \rightarrow a_{1,0}$ and $M \rightarrow M_{0}$ with $0<a_{1,0}<\infty$ and $0<$ $M_{0}<\infty$ implying $a_{2} \rightarrow 0$ and $a_{3} \rightarrow 0$, the ellipsoids degenerate to a rod. Then the velocity field of Equation (17) always diverges logarithmically, which follows from Equations (9). In fact, two anti-parallel, non-interacting streams in the $x^{1}$ direction with infinite velocity emerge such that the solution is static. The same holds true for the case $a_{1,0}=\infty$. In these cases, the global solution is not admissible for a $\mathrm{PN}$ approximation sufficiently close to the limit $\bar{a}_{2}=0$. However, if $M_{0}=0$ is approached sufficiently fast, the limit describes a Newtonian solution that can be interpreted as two anti-parallel streams of massless particles with a finite velocity. Hence, a PN approximation might be possible. We present the details of this in Appendix C

If $a_{1,0}=0$ and $0<M_{0}<\infty$, we obtain again a point mass. If the limit of $-a_{1} \ln \bar{a}_{3}$ is sufficiently small for $\bar{a}_{2} \rightarrow 0$, then the velocity is subluminal during the limiting process.

\subsection{The exterior solution}

We describe here the formalism with which the exterior solution of the $1 \mathrm{PN}$ equilibrium figures is obtained in closed form in Section 3. As a practical example, we apply the algorithm to the Newtonian Dedekind ellipsoids. We introduce ellipsoidal coordinates $\lambda^{i}$ with $\lambda^{1}>k>\lambda^{2}>h>\lambda^{3}>0$ with

\footnotetext{
${ }^{2}$ We make use of the common Landau notation, where for two functions $f(x)$ and $g(x)$ we have $f \in o_{x}(g)$, if $\lim _{x \rightarrow 0} \frac{f(x)}{g(x)}=0$. If the dependent variable is clear from context we drop the index of the Landau symbol.
} 
$h^{2}=a_{1}^{2}-a_{2}^{2}$ and $k^{2}=a_{1}^{2}-a_{3}^{2}$, see e.g. Byerly (1893):

$$
\begin{aligned}
& \left(x^{1}\right)^{2}=\frac{\left(\lambda^{1}\right)^{2}\left(\lambda^{2}\right)^{2}\left(\lambda^{3}\right)^{2}}{h^{2} k^{2}}, \\
& \left(x^{2}\right)^{2}=-\frac{1}{h^{2}\left(k^{2}-h^{2}\right)} \prod_{i=1}^{3}\left(\left(\lambda^{i}\right)^{2}-h^{2}\right), \\
& \left(x^{3}\right)^{2}=\frac{1}{h^{2}\left(k^{2}-h^{2}\right)} \prod_{i=1}^{3}\left(\left(\lambda^{i}\right)^{2}-k^{2}\right) .
\end{aligned}
$$

These coordinates cover the octant $x^{i}>0$, which is sufficient since the problem is reflection-symmetric with respect to the surfaces $x^{i}=0$. We assume that the PN configuration has this symmetry as well. The surface of the ellipsoid is characterized by $\lambda^{1}=a_{1}$. Using these coordinates, a separation of variables in the Poisson equation with a density $g$ the support of which is an ellipsoid is possible. The solution $f$ of $\Delta f=-4 \pi g$ will be of the form

$$
\sum_{n=0}^{\infty} \sum_{m=1}^{2 n+1} f_{m}^{n}\left(\lambda^{1}\right) E_{m}^{n}\left(\lambda^{2}\right) E_{m}^{n}\left(\lambda^{3}\right),
$$

where the functions $E_{m}^{n}$ are the Lamé functions of the first kind. Their definition and the first few members of this complete set of functions can be found in Bverly (1893). The function $f_{m}^{n}$ will be obtained as a solution of the inhomogeneous Lamé equation $L_{m}^{n}\left(\lambda^{1}\right)[f]=\tilde{g}_{m}^{n}\left(\lambda^{1}\right)$, where the Lamé operator $L_{m}^{n}$ is given by

$$
\begin{aligned}
& L_{m}^{n}(x)=\left(x^{2}-h^{2}\right)\left(x^{2}-k^{2}\right) \frac{d^{2}}{d x^{2}}+ \\
& \quad x\left(2 x^{2}-h^{2}-k^{2}\right) \frac{d}{d x}+\left(K_{m}^{n}-n(n+1) x^{2}\right) .
\end{aligned}
$$

The characteristic values $K_{m}^{n}$ of the Lamé functions are also defined in Bverly (1893). The $\tilde{g}_{m}^{n}\left(\lambda^{1}\right)$ are the expansion coefficients of the density $\mu$ with respect to the ellipsoidal harmonics:

$$
\begin{aligned}
& \left(\left(\lambda^{1}\right)^{2}-\left(\lambda^{2}\right)^{2}\right)\left(\left(\lambda^{1}\right)^{2}-\left(\lambda^{3}\right)^{2}\right) g\left(\lambda^{i}\right) \\
& =\sum_{n=0}^{\infty} \sum_{m=1}^{2 n+1} \tilde{g}_{m}^{n}\left(\lambda^{1}\right) E_{m}^{n}\left(\lambda^{2}\right) E_{m}^{n}\left(\lambda^{3}\right) .
\end{aligned}
$$

Since $g$ vanishes outside of the ellipsoid, the equation becomes homogeneous and the sole solution with the correct asymptotics is given by

$$
f_{m}^{n}\left(\lambda^{1}\right)=C_{m}^{n} F_{m}^{n}\left(\lambda^{1}\right),
$$

where $F_{m}^{n}$ denotes the Lamé functions of the second kind, see again Byerly (1893). These follow from the Lamé functions of the first kind via

$$
\begin{aligned}
& F_{m}^{n}(x)=E_{m}^{n}(x) \times \\
& \int_{x}^{\infty}\left(E_{m}^{n}(u)\right)^{-2}\left(\left(u^{2}-h^{2}\right)\left(u^{2}-k^{2}\right)\right)^{-\frac{1}{2}} d u .
\end{aligned}
$$

To the orders, which appear in the present paper, the $F_{m}^{n}$ can be given explicitly in closed form in terms of elliptic functions.

In general, we have to solve the inhomogeneous Lamé equation. However, we can rely for all potentials that we have to calculate on a result by Ferrers (1877). There the interior solution of the Poisson equation with a density that is polynomial in Cartesian coordinates inside an ellipsoid is given. It is also established that this solution can be connected to an exterior solution, which vanishes at infinity, such that the solution is continuously differentiable everywhere. Thus, we obtain $f_{m}^{n}\left(\lambda^{1}\right)$ for $\lambda^{1}<a_{1}$ simply by a coordinate transformation of Ferrers' interior solution from Cartesian to ellipsoidal coordinates. Since the interior solution is polynomial in Cartesian coordinates, the expansion of this in ellipsoidal surface harmonics $E_{m}^{n}\left(\lambda^{2}\right) E_{m}^{n}\left(\lambda^{3}\right)$ terminates at finite order and we can simply read the $f_{m}^{n}$ off. Hence, $C_{m}^{n}$ can afterwards be obtained by

$$
C_{m}^{n}=f_{m}^{n}\left(a_{1}\right) F_{m}^{n}\left(a_{1}\right)^{-1}
$$

and the potential $U$ is completely determined.

We illustrate this method for the Dedekind ellipsoids, which are of the type considered by Ferrers (1877). The interior solution can be written after a transformation to ellipsoidal coordinates as

$$
\begin{aligned}
U= & f_{1}^{0}\left(\lambda^{1}\right) E_{1}^{0}\left(\lambda^{2}\right) E_{1}^{0}\left(\lambda^{3}\right)+ \\
& \sum_{i=1}^{2} f_{i}^{2}\left(\lambda^{1}\right) E_{i}^{2}\left(\lambda^{2}\right) E_{i}^{2}\left(\lambda^{3}\right), \\
f_{1}^{0}\left(\lambda^{1}\right)= & -\frac{4}{3} \pi G \mu\left(3\left(\lambda^{1}\right)^{4}-\right. \\
& \left.2\left(\lambda^{1}\right)^{2}\left(h^{2}+k^{2}\right)+h^{2} k^{2}\right),
\end{aligned}
$$




$$
f_{1 / 2}^{2}\left(\lambda^{1}\right)=-2 \pi G \mu\left(1 \pm \frac{3\left(\lambda^{1}\right)^{2}-h^{2}-k^{2}}{\sqrt{h^{4}-h^{2} k^{2}+k^{4}}}\right) .
$$

This implies that the exterior solution has the form

$$
\begin{aligned}
U= & C_{1}^{0} F_{1}^{0}\left(\lambda^{1}\right) E_{1}^{0}\left(\lambda^{2}\right) E_{1}^{0}\left(\lambda^{3}\right)+ \\
& \sum_{i=1}^{2} C_{i}^{2} F_{i}^{2}\left(\lambda^{1}\right) E_{i}^{2}\left(\lambda^{2}\right) E_{i}^{2}\left(\lambda^{3}\right),
\end{aligned}
$$

where the constants $C_{m}^{n}$ are obtained from Equations (16) and (17).

In fact, this procedure allows us to obtain the exterior solution for mass densities of the form $\mu_{i j k}=$ const. $\left(x^{1}\right)^{i}\left(x^{2}\right)^{j}\left(x^{3}\right)^{k}$ in closed form. This enables us to determine the $1 \mathrm{PN}$ metric in the exterior region in closed form for the Dedekind ellipsoids as well as the Jacobi ellipsoids in exactly the same way. Higher order ellipsoidal harmonics will be necessary in this scheme. However, the calculations are tedious and will not be presented in detail here. We will only repeat the form of the higher moments in the interior of the ellipsoid, which are the starting point for the straightforward calculations, in Appendix $\mathrm{A}$.

\section{The $1 \mathrm{PN}$ approach}

In Chandrasekhar (1965b), a set of field equations was discussed whose solutions describe perfect fluids dynamically to first order in $\frac{1}{c^{2}}$ in general relativity. In a subsequent series of papers (Chandrasekhar 1965a, 1967a, b, 1971b,a; Chandrasekhar \& Elbert 1974), solutions to these equations were constructed using different Newtonian configuration as a starting point- namely Maclaurin ellipsoids, Jacobi ellipsoids and Dedekind ellipsoids. Although these are all equilibrium figures, the field equations in Chandrasekhar (1965b) allow for non-stationary solutions, too. Thus, one always has to determine and to solve the equations belonging to the dynamical aspects of the fluid. In contrast, the projection formalism described in Geroch (1971) and the field equations derived therein implement the stationarity from the beginning and can be used only in the description of equilibrium figures. In our case, where we are primarily interested in stationary solutions the latter equations are more advantageous especially if one goes to higher PN orders. However, to the $1 \mathrm{PN}$ order both approaches yield the same result in the case of stationarity; discrepancies will become apparent only at higher orders. For equations describing higher order PN corrections, see, e.g., Chandrasekhar \& Nutku (1969); Asada \& Shibata (1996); Asada et al. (1996).

We use the same expansion of the metric as in Chandrasekhar (1965b):

$$
\begin{aligned}
g_{\alpha \beta} & =-\left(1+\frac{2 U}{c^{2}}\right) \delta_{\alpha \beta}, \\
g_{\alpha 0} & =4 U_{\alpha}^{(3)} c^{-3}, \\
g_{00} & =1-2 U c^{-2}+2\left(U^{2}-\delta U-2 \Phi\right) c^{-4},
\end{aligned}
$$

where Greek indices run from 1 to $3, \delta_{\alpha \beta}$ denotes the Kronecker delta, $x^{0}=c t$. $U$ is the Newtonian gravitational potential, which we assume here to be that of a Dedekind ellipsoid as discussed in Section 2 The contribution $\delta U$ is defined momentarily.

The shape of the PN configuration is no longer that of the Newtonian ellipsoid $S^{(0)}$ (cf. Equation (21) and we denote it by

$$
S=S^{(0)}+S^{(2)} c^{-2} .
$$

Let us introduce a potential $U^{\prime}$ that is a solution of the Poisson equation (11) for this perturbed ellipsoid, i.e.,

$$
\Delta U^{\prime}=-4 \pi G \mu
$$

with $\mu=$ const. for $S<0$. The symbol $\delta U$ in Equation (19) is then defined by

$$
\delta U=\left(U^{\prime}-U\right) c^{2} .
$$

Note that we use the expansion parameter $c^{-1}$ to retain the compatibility with Papers I and II. However, one could transform the results easily to a more physical expansion parameter, e.g., $\varepsilon^{2}=\frac{2 M G}{c^{2} a_{1}}$.

The pressure and the velocity field are also expanded

$$
p=p^{(0)}+p^{(2)} c^{-2}, \quad v^{\alpha}=v^{(0) \alpha}+v^{(2) \alpha} c^{-2},
$$

where $v^{(0) \alpha}$ is the Newtonian velocity $\sqrt{3}$ and $p^{(0)}$ is the Newtonian pressure, cf. Equations (8). We

\footnotetext{
${ }^{3}$ The three-velocity $v^{\alpha}$ is defined as in Paper II and does not refer to the spatial components of the four-velocity $u^{i}=$ $\frac{d x^{i}}{d \tau}$, but is instead defined as $v^{\alpha}=\frac{d x^{\alpha}}{d t}=c \frac{u^{\alpha}}{u^{0}}$.
} 
assume that the homogeneous mass density does not change to any $\mathrm{PN}$ order.

The $1 \mathrm{PN}$ equations of a self-gravitating perfect fluid read4, see Chandrasekhar (1965b),

$$
\begin{aligned}
& \Delta \Phi=-4 \pi G \mu\left(v^{(0)^{2}}+\frac{3 p^{(0)}}{2 \mu}+U\right), \\
& \Delta U_{\alpha}^{(3)}=-4 \pi G \mu v_{\alpha}^{(0)}, \\
& v_{, \alpha}^{(2) \alpha}=-\left(v^{(0)^{2}}+\frac{p^{(0)}}{\mu}+2 U\right)_{, \alpha} v^{(0) \alpha}, \quad(24) \\
& \frac{p_{, \alpha}^{(2)}}{\mu}=\left(\delta U+2 \Phi+2 v^{(0)^{2}} U+\frac{p^{(0)^{2}}}{2 \mu^{2}}\right)_{, \alpha}- \\
& 2 U^{(0)} v^{(0)^{2}}{ }_{, \alpha}+4 v^{(0) \beta}\left(U_{\alpha, \beta}-U_{\beta, \alpha}\right)+ \\
& v^{(0) \beta}\left(\left(v^{(0)^{2}}+4 U\right) v^{(0)}{ }_{\alpha, \beta}+{ }_{\alpha, \beta}-v^{(2) \beta} v^{(0)}{ }_{\alpha, \beta},\right. \\
&\left.4 v_{\alpha}^{(0)} U_{, \beta}\right)-v^{(0) \beta} v^{(2)}{ }_{\alpha,}
\end{aligned}
$$

where $v^{(0)}{ }^{2}$ is the square of the Newtonian velocity field. Since the spatial part of the metric is easily obtained and already incorporated in Equation (19) the sole equations that remain to be solved in order to obtain the metric to $1 \mathrm{PN}$ are the first two and the one which determines $\delta U$. The other equations determine the $1 \mathrm{PN}$ corrections to the pressure, velocity field and the surface.

\subsection{The velocity field and the surface}

For the $1 \mathrm{PN}$ correction of the surface we choose the following ansatz

$$
\begin{gathered}
S^{(2)}=2 \pi G \mu\left(a_{1}^{2} \sum_{i=1}^{2} S_{i}\left(\left(\frac{x^{i}}{a_{i}}\right)^{2}-\left(\frac{x^{3}}{a_{3}}\right)^{2}\right)+\right. \\
S_{3}\left(x^{1}\right)^{2}\left(\frac{1}{3}\left(\frac{x^{1}}{a_{1}}\right)^{2}-\left(\frac{x^{2}}{a_{2}}\right)^{2}\right)+ \\
S_{4}\left(x^{2}\right)^{2}\left(\frac{1}{3}\left(\frac{x^{2}}{a_{2}}\right)^{2}-\left(\frac{x^{3}}{a_{3}}\right)^{2}\right)+ \\
\left.S_{5}\left(x^{3}\right)^{2}\left(\frac{1}{3}\left(\frac{x^{3}}{a_{3}}\right)^{2}-\left(\frac{x^{1}}{a_{1}}\right)^{2}\right)\right)
\end{gathered}
$$

\footnotetext{
${ }^{4}$ Indices are raised and lowered in these equations with the flat Euclidean metric.
}

and for the velocity

$$
\begin{gathered}
\frac{v_{1}^{(2)}}{(\pi G \mu)^{\frac{3}{2}}}=x^{2}\left(a_{1}^{2} w_{1}+\hat{q}_{1}\left(x^{1}\right)^{2}+\right. \\
\left.r_{1}\left(x^{2}\right)^{2}+t_{1}\left(x^{3}\right)^{2}\right), \\
\frac{v_{2}^{(2)}}{(\pi G \mu)^{\frac{3}{2}}}=x^{1}\left(a_{2}^{2} w_{2}+\hat{q}_{2}\left(x^{2}\right)^{2}+\right. \\
\left.r_{2}\left(x^{1}\right)^{2}+t_{2}\left(x^{3}\right)^{2}\right), \\
\frac{v_{3}^{(2)}}{(\pi G \mu)^{\frac{3}{2}}}=q_{3} x^{1} x^{2} x^{3} .
\end{gathered}
$$

In Paper II, both, $w_{1}$ and $w_{2}$, were not considered. In Gürlebeck \& Petroff (2010), we showed that these linear contributions can be used to allow a rigidly rotating axisymmetric limit of the 1 PN Dedekind ellipsoids coinciding with the $1 \mathrm{PN}$ Maclaurin ellipsoids. Moreover, it seems natural to allow a $1 \mathrm{PN}$ contribution to the Newtonian constant $\Omega$ analogous to the angular velocity in the case of rigid rotation, see Papers I. Furthermore, we introduced the constant $\hat{q}_{1}=q_{1}+q$ and $\hat{q}_{2}=q_{2}-q$ compared to Paper II. The ansatz for the surface is the same as in Paper I, i.e., it originates from a Lagrangian displacement of all fluid elements such that the coordinate volume remains constant. The Lagrangian displacement reads, cf. Equation (41) in Paper II,

$$
\begin{aligned}
& \xi_{\mu}=\frac{\pi G \mu a_{1}^{2}}{c^{2}} \sum_{A=1}^{5} S_{A} \xi_{\mu}^{A} \\
& \left(\xi_{\mu}^{1}\right)=\left(x^{1}, 0,-x^{3}\right), \quad\left(\xi_{\mu}^{2}\right)=\left(0, x^{2},-x^{3}\right) \\
& \left(\xi_{\mu}^{3}\right)=\frac{1}{3 a_{1}^{2}}\left(\left(x^{1}\right)^{3},-3\left(x^{1}\right)^{2} x^{2}, 0\right) \\
& \left(\xi_{\mu}^{4}\right)=\frac{1}{3 a_{1}^{2}}\left(0,\left(x^{2}\right)^{3},-3\left(x^{2}\right)^{2} x^{3}\right) \\
& \left(\xi_{\mu}^{5}\right)=\frac{1}{3 a_{1}^{2}}\left(-3\left(x^{3}\right)^{2} x^{1}, 0,\left(x^{3}\right)^{3}\right)
\end{aligned}
$$

As was pointed out in Bardeen (1971), it is more physical to fix parameters with an immediate physical interpretation like the mass instead the coordinate volume. However, by following $\mathrm{Pa}-$ per II, it is easier to compare our results with theirs. As it was discussed in Chandrasekhar (1971b) and in Paper II, an arbitrary contribution to the coordinate volume can be achieved 
by an additional displacement of the form $\xi_{6}=$ $\pi G \mu a_{1}^{2} c^{-2} S_{6}\left(x^{1}, x^{2}, x^{3}\right)$, whose divergence does not vanish. Alternatively, one can arrive at the same result by considering a different underlying Newtonian configuration, i.e. by substituting in our final results $a_{1}$ by $a_{1}+2 \pi G \mu a_{1}^{2} c^{-2} S_{6}$ while keeping $\bar{a}_{2}$ and $\bar{a}_{3}$ fixed.

Note that higher order polynomials could also be allowed in the ansatz (25), which would eventually lead to a homogeneous system for their coefficients. The form of the solution we use here is the minimal one needed to satisfy the inhomogeneous equations and get a configuration that is Dedekind-like in the sense discussed in Section 1]

\subsection{The solution for the metric functions}

In this section, we will repeat the interior solution for the metric components to an extent that is necessary to construct the exterior solution as well. Since the changes in the ansatz (25) do not affect Equations (24a and (24b) their solutions are the same as obtained in Paper II. The equations are of the type treated in Ferrers (1877), namely they have a polynomial density due to the form of the Newtonian solution (8). Thus, they can be expressed in terms of the higher moments of the homogeneous density $D_{i_{1} i_{2} \ldots}$, cf. Appendix A. With these, the solutions to Equations (24a) and (24b) read

$$
\begin{aligned}
\Phi & =\pi G \mu\left[U\left(\frac{3}{2} a_{3}^{2} A_{3}+A_{\emptyset}\right)-\frac{5}{2} A_{3} D_{33}\right. \\
& -\left(A_{1}+\frac{3}{2} \bar{a}_{3}^{2} A_{3}-\bar{a}_{2}^{2} \bar{\Omega}^{2}\right) D_{11} \\
& \left.-\left(A_{2}+\frac{3 \bar{a}_{3}^{2}}{2 \bar{a}_{2}^{2}} A_{3}-\frac{1}{\bar{a}_{2}^{2}} \bar{\Omega}^{2}\right) D_{22}\right] \\
U_{1} & =-\frac{a_{1}}{a_{2}} \Omega D_{2}, \quad U_{2}=\frac{a_{2}}{a_{1}} \Omega D_{1} \\
U_{3} & =0 .
\end{aligned}
$$

A similar approach can be taken for $\delta U$. It can be written as

$$
\frac{\delta U}{c^{2}}=-G \frac{\partial}{\partial x^{\mu}} \int \frac{\mu \xi^{\mu}}{\left|\vec{x}-\vec{x}^{\prime}\right|} d^{3} x^{\prime},
$$

cf. Equation (58) in Chandrasekhar (1967a), i.e., as the sum of derivatives of Poisson integrals. As such $\delta U$ is not continuously differentiable across the surface, which is a problem inherent to this coordinate system. Using surface adapted Cartesian coordinates $y^{\mu}=x^{\mu}+\xi^{\mu}$ the metric components are continuously differentiable. But we do not use them here but rather follow Paper II.

The densities in the Poisson integrals (28) are polynomial for the Lagrangian displacement $\xi^{\alpha}$ (26). Thus, the algorithm described in Section 2.2 is applicable. Taking the derivatives afterwards yields

$$
\begin{aligned}
& \delta U=-\mu^{2} G^{2} \pi\left(S_{1} a_{1}^{2}\left(D_{1,1}-D_{3,3}\right)+\right. \\
& S_{2} a_{1}^{2}\left(D_{2,2}-D_{3,3}\right)+\frac{S_{3}}{3}\left(D_{111,1}-3 D_{112,2}\right)+ \\
& \left.\frac{S_{4}}{3}\left(D_{222,2}-3 D_{223,3}\right)+\frac{S_{5}}{3}\left(D_{333,3}-3 D_{331,1}\right)\right) .
\end{aligned}
$$

Note that only six of the ten third order moments $D_{i j k}$ and only the diagonal terms of the second order moments $D_{i j}$ are necessary, cf. Equation (27). In order to fix the constants $S_{i}$, we solve the Bianchi identity in Equation (24) in the next section.

\subsection{Corrections to the pressure, the ve- locity field, and the surface}

Although the changes in the ansatz will not change the calculations fundamentally, we will describe it in more detail and give also intermediate solutions and analytic expressions. The main reason is that we were unable to reproduce the numerical data given in Paper II for the case $w_{1}=$ $w_{2}=0$ (see the discussion in Gürlebeck \& Petroff (2010) for further details). Thus, it might prove helpful for rectifying this discrepancy or at the very least make our calculations repeatable. The formulae, which are too lengthy for the text here, can be found in Appendix B.

Inserting our ansatz in the integrability condition for the gradient of the pressure, i.e. Equation (24d), and using the polynomial structure for a comparison of coefficients yields the following solution:

$$
\begin{aligned}
t_{2} & =\bar{a}_{2}^{2} t_{1}, \\
r_{2} & =4\left(\bar{a}_{2}^{3}-\bar{a}_{2}\right) \bar{\Omega}\left(\bar{B}_{112}+\bar{B}_{122}\right)+\left(\frac{1}{\bar{a}_{2}}-\bar{a}_{2}^{3}\right) \bar{\Omega}^{3} \\
& +\frac{1}{3}\left(\hat{q}_{1}\left(\bar{a}_{2}^{2}+2\right)+\hat{q}_{2}\left(2 \bar{a}_{2}^{2}+1\right)-3 r_{1} \bar{a}_{2}^{2}\right), \\
q_{3} & =-4\left(\bar{a}_{2}-\frac{1}{\bar{a}_{2}}\right) \bar{\Omega}\left(\bar{B}_{123}+\bar{B}_{13}\right) .
\end{aligned}
$$


Thus, the constant $q_{3}$ is already determined completely and independently of the parameters $w_{i}$.

Repeating the same for the continuity Equation (24c) and using the results (30) gives further constraints on the constants

$$
\begin{aligned}
\hat{q}_{2} & =2\left(\bar{a}_{2}-\frac{1}{\bar{a}_{2}}\right) \bar{\Omega}\left(\bar{a}_{2}^{2} \bar{B}_{123}+\bar{B}_{23}\right) \\
& +2\left(\bar{a}_{2}-\frac{1}{\bar{a}_{2}}\right) \bar{\Omega}^{3}-\hat{q}_{1} .
\end{aligned}
$$

A further simplification is achieved by requiring the necessary condition that the normal component of the velocity vanishes at the surface up to $1 \mathrm{PN}$. This gives

$$
\begin{aligned}
r_{1} & =\frac{1}{\bar{a}_{2}^{3}}\left[\overline { \Omega } \left(\frac { 1 } { 3 ( \overline { a } _ { 2 } ^ { 2 } - 1 ) } \left[3\left(5 \bar{a}_{2}^{2}+1\right)\left(S_{2}-S_{1}\right)\right.\right.\right. \\
& \left.-3\left(3 \bar{a}_{2}^{2}-1\right) S_{3}+2 \bar{a}_{2}^{2}\left(2 \bar{a}_{2}^{2}+1\right) S_{4}\right] \\
& -6 \bar{a}_{2}^{2}\left(\bar{B}_{112}+\bar{B}_{122}\right) \\
& \left.-2\left(2 \bar{a}_{2}^{2}+1\right)\left(\bar{a}_{2}^{2} \bar{B}_{123}+\bar{B}_{23}\right)\right) \\
& \left.-\frac{\left(w_{1}+w_{2}\right) \bar{a}_{2}\left(5 \bar{a}_{2}^{2}+1\right)}{2\left(\bar{a}_{2}^{2}-1\right)}-\frac{\left.\left(5 \bar{a}_{2}^{2}+1\right) \bar{\Omega}^{3}\right]}{2}\right] \\
t_{1}= & \frac{1}{2 \bar{a}_{2} \bar{a}_{3}^{2}}\left[2 \overline { \Omega } ^ { 2 } \left(S_{2}-S_{1}-\bar{a}_{2}^{2} S_{4}+\bar{a}_{3}^{2} S_{5}\right.\right. \\
& \left.\left.+2\left(\bar{a}_{2}^{2}-1\right)\left(\bar{B}_{123}+\bar{B}_{13}\right)\right)-\bar{a}_{2}\left(w_{1}+w_{2}\right)\right] \\
\hat{q}_{1}= & \bar{\Omega}\left(\frac { 1 } { \overline { a } _ { 2 } ( \overline { a } _ { 2 } ^ { 2 } - 1 ) } \left[3\left(\bar{a}_{2}^{2}+1\right)\left(S_{2}-S_{1}\right)\right.\right. \\
& \left.-\left(5 \bar{a}_{2}^{2}-3\right) S_{3}+2 \bar{a}_{2}^{2} S_{4}\right]-6 \bar{a}_{2}\left(\bar{B}_{112}+\bar{B}_{122}\right) \\
& \left.-2 \frac{\left(\bar{a}_{2}^{2}+2\right)}{\bar{a}_{2}}\left(\bar{a}_{2}^{2} \bar{B}_{123}+\bar{B}_{23}\right)\right) \\
& -\frac{3\left(w_{1}+w_{2}\right)\left(\bar{a}_{2}^{2}+1\right)}{2\left(\bar{a}_{2}^{2}-1\right)}-\frac{\left(\bar{a}_{2}^{2}+5\right) \bar{\Omega}^{3}}{2 \bar{a}_{2}} .
\end{aligned}
$$

Up to now, all coefficients entering the $1 \mathrm{PN}$ corrections to the velocity field can be given in terms of $w_{i}$ and $S_{i}$. To determine the surface coefficients $S_{i}$, we have to impose that the pressure vanishes at the surface up to first PN order, which leads to a linear system of equations:

$$
\sum_{j=0}^{5} M_{i j} S_{j}=b_{i}^{(0)}+b_{i}^{(1)} w_{1}+b_{i}^{(2)} w_{2}=b_{i} .
$$

We give the analytic and lengthy expressions of the coefficient matrix $\left(M_{i j}\right)$ and the inhomogeneity $\left(b_{i}\right)$ in Appendix B.2 The equations for $i=$ $1, \ldots, 5$ ensure that the pressure at the surface is constant. Having solved those, the PN contribution to the central pressure $p_{C}^{(2)}=S_{0} a_{1}^{4} \mu(\mu G)^{2}$ is obtained using the equation for $i=0$ such that the pressure vanishes at the surface. The parameters $S_{i}, t_{i}, r_{i}$ and $q_{i}$ are plotted along the $1 \mathrm{PN}$ Dedekind sequence in Figure 2 in Appendix D using a parameterization described in Section 3.4.2

The singularity which was discovered in Paper II has its origin in a vanishing determinant of the coefficient matrix $\left(M_{i j}\right)$. How this singularity can be removed is discussed in Section 3.4.2. A solution $S_{i}$ of Equation (33) depends on the $w_{i}$. Hence, all constants entering our ansatz (25) but $q_{3}$ are obtained in terms of the $w_{i}$. The only requirement for the choice of the $w_{i}$ is that we have a Dedekindlike configuration in the sense of Section 1 and that the resulting surface (25) is still closed. The latter is just a reformulation of the fact that the $1 \mathrm{PN}$ corrections must be small compared to the Newtonian quantities though offering here an explicit and necessary criterion.

\subsection{Properties of the solution}

In this section, we discuss some properties of the family of solutions described in Sections 3.2 and 3.3. The $w_{i}$ can be chosen independently for each $\bar{a}_{2}$ along the family so that two free functions $w_{i}\left(\bar{a}_{2}\right)$ remain. We assume here continuous functions $w_{i}$ in order to ensure a continuous family of 1 PN Dedekind ellipsoids. In Section 3.4.2 we determine the conditions for the $w_{i}$ imposed by the requirement that the $1 \mathrm{PN}$ Dedekind ellipsoids have similar properties as in the Newtonian case. We treated one of these requirements in Gürlebeck \& Petroff (2010), which ensures that the 1 PN Dedekind ellipsoids are axially symmetric and rigidly rotating in the limit $\bar{a}_{2} \rightarrow 1$ coinciding with the $1 \mathrm{PN}$ Maclaurin ellipsoids. This is achieved if $w_{1}(1)=-w_{2}(1)$ holds in the limit. Analytic expressions can be found in that paper. Other restrictions do not ensue from this property.

\subsubsection{The mass and the angular momentum}

Let us first characterize the $1 \mathrm{PN}$ corrections by two physical parameters of the Dedekind ellipsoids 
- the mass and the angular momentum. According to the definition in Chandrasekhar \& Nutku (1969), the 1 PN perturbation of the conserved $\operatorname{mass} M^{(2)}$ is

$$
M^{(2)}=M^{(0)} \frac{\pi}{5} G \mu a_{1}^{2}\left(12 \bar{A}_{\emptyset}+\left(1+\bar{a}_{2}^{2}\right) \bar{B}_{12}\right) .
$$

This is independent of the choice of $w_{i}$. The angular momentum of the Newtonian solution as well as the $1 \mathrm{PN}$ Dedekind ellipsoids points in the $x^{3}$ direction. The first evaluates to

$$
L^{(0)}=\frac{8 \pi}{15} \mu \Omega a_{1}^{2} a_{2}^{2} a_{3},
$$

whereas the latter is rather lengthy and is shown in Appendix B.1. Here, we only present the plot, cf. Figure1 $L^{(2)}$ is linear in the $w_{i}$ as are all other quantities, which we investigate. Thus, we depict for all constants, say $L^{(2)}$, the coefficients in front of the $w_{i}$, i.e., $L^{(2)}=L_{0}^{(2)}+L_{1}^{(2)} w_{1}+L_{2}^{(2)} w_{2}$. We use a solid line for $L_{0}^{(2)}$, a dashed line for $L_{1}^{(2)}$ and a dot-dashed line for $L_{2}^{(2)}$. The part $L_{0}^{(2)}$ equals the respective constants in Paper II, though as discussed in length in Gürlebeck \& Petroff (2010) the numerical values do not agree.

\subsubsection{The singularity}

The singularity at $\bar{a}_{2}^{s}=0.33700003168 \ldots$. cf. Figure 1(b) is the one already discovered in $\mathrm{Pa}$ per II. The importance of singularities in $1 \mathrm{PN}$ approximations of equilibrium figures and the issues with the 1 PN Dedekind sequence of that paper was discussed in Section 1. Since we are able to introduce singularities in our $1 \mathrm{PN}$ Dedekind solutions at arbitrary points $\bar{a}_{2}$ via $w_{i}\left(\bar{a}_{2}\right)$, it is obvious that not all singularities are necessarily at physically distinguished points. That we can use the $w_{i}\left(\bar{a}_{2}\right)$ to remove the singularity at $\bar{a}_{2}^{S}$ for all physical quantities is shown in this section.

Evaluating the determinant of the coefficient matrix $\left(M_{i j}\right)$, cf. Equation (33) and Appendix B.2, numerically shows that it changes sign at $\bar{a}_{2}^{s}$. One of the eigenvalues, say $\lambda$, of the coefficient matrix $\left(M_{j i}\right)$ vanishes there. Multiplying Equation (33) with the eigenvector $\left(\beta_{i}\right)$ to the eigenvalue $\lambda$

\footnotetext{
${ }^{5}$ Subsequently, we truncate numerical values to six digits.
}

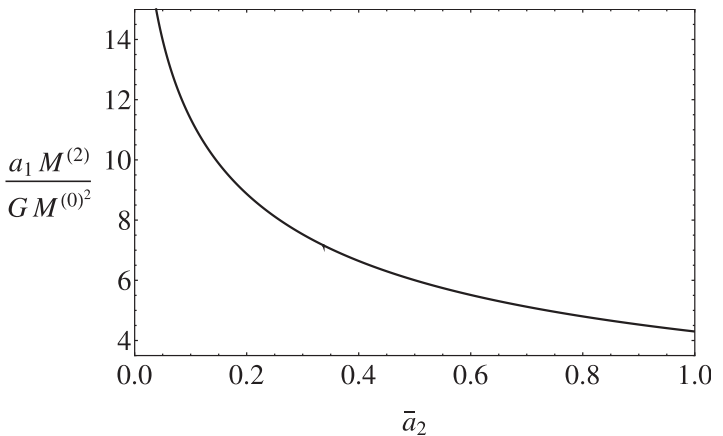

(a)

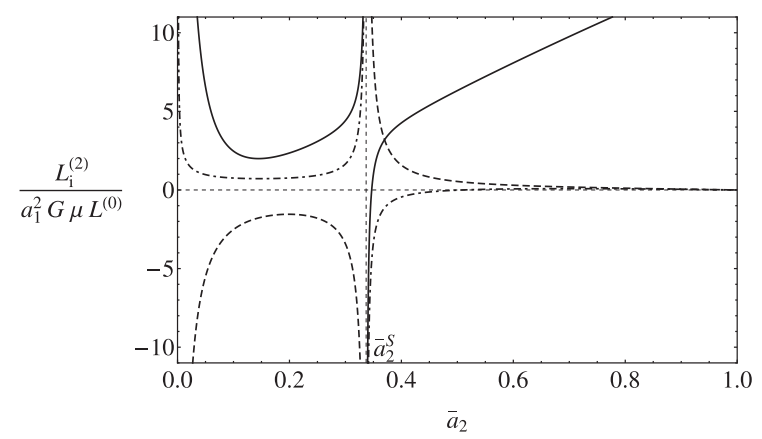

(b)

Fig. 1. - $1 \mathrm{PN}$ corrections to (a) the mass and (b) the angular momentum.

of the transposed matrix yields the condition

$$
\begin{aligned}
& \sum_{i=1}^{3} \beta_{i} b_{i}= \\
& 0.083600-0.235534 w_{1}+0.099994 w_{2}=0 .
\end{aligned}
$$

In Paper II, whose results are obtained by setting $w_{1}=w_{2}=0$, Equation (36) could not be satisfied. Hence, a singularity is inevitable there in lieu of our approach. We have a more general inhomogeneity in Equation (33), cf. the $b_{i}^{1 / 2}$. Choosing at $\bar{a}_{2}^{s}$

$$
w_{1}^{s}=0.354937+0.424544 w_{2},
$$

Equation (36) is identically satisfied and the remaining system of four equations in Equations (33) is regular. To ensure that this holds in the limit $\bar{a}_{2} \rightarrow \bar{a}_{2}^{s}$ as well, higher orders have to be taken into account. However, as one can readily check numerically, the equation $\sum_{i, j} \frac{\beta_{i} M_{i j} S_{j}}{\lambda}=$ $\sum_{i} \frac{\beta_{i} b_{i}}{\lambda}$ is well-defined in the limit $\bar{a}_{2} \rightarrow \bar{a}_{2}^{s}$ if 
$w_{1}-w_{1}^{s} \in O(\lambda)$. Thus, this equation can be used instead of, for instance, $\sum_{j} M_{1 j} S_{j}=b_{1}$. The resulting coefficient matrix is now regular and the solution finite. Let us introduce instead of $w_{1}$ a new parameter defined by $w_{1}=w_{1}^{s}\left(w_{2}\right)+\lambda \hat{w}_{1}$, where $\hat{w}_{1}$ and $w_{2}$ are arbitrary. Then, the surface coefficients at $\bar{a}_{2}^{s}$ evaluate to

$$
\begin{aligned}
& S_{1}^{s}=0.136453-0.243073 w_{2}+0.054186 \hat{w}_{1}, \\
& S_{2}^{s}=-0.195876+0.154884 w_{2}-0.044769 \hat{w}_{1}, \\
& S_{3}^{s}=-0.119902+0.212999 w_{2}+0.221751 \hat{w}_{1}, \\
& S_{4}^{s}=-1.393285+0.412379 w_{2}+0.429324 \hat{w}_{1}, \\
& S_{5}^{s}=4.761466-1.470110 w_{2}-1.530518 \hat{w}_{1} .
\end{aligned}
$$

Since these parameters enter the velocity field linearly and their coefficients in Equations (30)-(32) are well-defined at $\bar{a}_{2}^{s}$, the entire solution is regular. Only Equation (37) is obtained as an extra condition for $\bar{a}_{2} \rightarrow \bar{a}_{2}^{s}$. As an example, the angular momentum reads under this constraint

$\frac{L_{s}^{(2)}}{a_{1}^{7} G^{\frac{3}{2}} \mu^{\frac{5}{2}}}=0.177158-0.005261 \hat{w}_{1}+0.009930 w_{2}$.

In contrast to Figure 1(b), this is finite. Equation (37) is not a good parametrization if $\bar{a}_{2} \rightarrow 0$ or $\bar{a}_{2} \rightarrow 1$. For the axially symmetric limit, a good parametrization close to $\bar{a}_{2}=1$ was discussed in Gürlebeck \& Petroff (2010) and the limit $\bar{a}_{2} \rightarrow$ 0 is treated in detail in Appendix C]. These two limits yield additional constraints for $w_{i}\left(\bar{a}_{2}\right)$ in the neighborhood of the respective points that can be satisfied simultaneously.

\subsubsection{The surface and the gravitomagnetic effect}

Which shapes can we expect for the $1 \mathrm{PN}$ Dedekind ellipsoids if no additional constraints are given? The gravitomagnetic effect, i.e., parallel matter streams repel each other, is already included in a $1 \mathrm{PN}$ approximation. Thus, one should find that the 1 PN Dedekind ellipsoid is elongated 6 in the $x^{3}$-direction compared to the Newtonian figure, since all the Newtonian streams are along ellipses in the same direction in parallel planes.

\footnotetext{
${ }^{6}$ Note that the qualitative picture does not change if one evaluates proper distances instead of coordinate distances as is done here for simplicity.
}

This qualitative argument is corroborated by the Maclaurin ellipsoids, which are also elongated in this direction close to the bifurcation point, see Petroff (2003a). Moreover, the matter streams in the $x^{2}$-direction for $x^{1}>0$ are all parallel and anti-parallel to those in $x^{1}<0$. The latter are on average at a larger distance. Thus, the repulsive effect should prevail and in this direction too we have an elongation. In the $x^{1}$-direction, a similar argument holds. Which effect is stronger, the repulsion in the $x^{2}$-direction or in the $x^{1}$-direction, depends on the choice of $w_{i}$. However, this conclusion disregards the pressure entirely and can only provide a rough idea for the resulting $1 \mathrm{PN}$ shape. More importantly, the Lagrangian displacement (26) yields a vanishing $1 \mathrm{PN}$ contribution to the coordinate volume and, thus, it does not allow for an elongation of the ellipsoid in all directions. In fact, we always observe elongations in the $x^{3}$ direction, and deformation in the other directions can be adjusted with different choices of the $w_{i}$. The three qualitatively different case are depicted in Figure 3 in Appendix D.

\subsubsection{The motion of the fluid}

To explicate the inner motion of the $1 \mathrm{PN}$ Dedekind ellipsoids, we integrated the velocity field of the fluid (25) numerically and discuss here the trajectories of a generic fluid element. In the Newtonian Dedekind ellipsoid, all fluid elements move along ellipses that are in planes with constant $x^{3}$. The time of revolution coincides for all fluid elements. In the $1 \mathrm{PN}$ approximation to these figures of equilibrium, the trajectories are distorted ellipses that are still closed and the motion is not any longer confined to planes with constant $x^{3}$. The latter follows directly from our ansatz in Equation (25b). The velocity in the $x^{3}$ direction vanishes at the coordinate planes so that no fluid element moves from the upper half of the 1 PN ellipsoid $\left(x^{3}>0\right)$ to the lower $\left(x^{3}<0\right)$ and vice versa. It also changes sign when the particle crosses the other coordinate planes. Moreover, the motion in the $x^{3}$-direction is periodic with half the time of revolution as period. Furthermore, the time of revolution of the fluid elements depends now on the starting point. The trajectories for particles at the surface, which stay there during their motion, are exemplary and can be inferred partially from Figure 3 
We gratefully acknowledge discussions with M. Ansorg, J. Bičák, J. Friedman and R. Meinel. NG was financially supported by the Grants No. GAUK 22708 and GAČR 205/09/H033 and DP by the Deutsche Forschungsgemeinschaft as part of the project "Gravitational Wave Astronomy" (SFB/TR7B1). Moreover, NG gratefully acknowledges support from the DFG within the Research Training Group 1620 "Models of Gravity".

\section{REFERENCES}

Asada, H., \& Shibata, M. 1996, Phys. Rev. D, 54, 4944

Asada, H., Shibata, M., \& Futamase, T. 1996, Prog. Theor. Phys., 96, 81

Bardeen, J. M. 1971, Astrophys. J., 167, 425

Byerly, W. E. 1893, An elementary treatise on Fourier series and spherical, cylindrical and ellipsoidal harmonics with application to problems in mathematical physics (Boston: Ginn and Company)

Chandrasekhar, S. 1965a, Astrophys. J., 142, 1513

—. 1965b, Astrophys. J., 142, 1488

—. 1967a, Astrophys. J., 147, 334

—. 1967b, Astrophys. J., 148, 621

—. 1970, Astrophys. J., 161, 571

—. 1971a, Astrophys. J., 167, 455

—. 1971b, Astrophys. J., 167, 447

—. 1987, Ellipsoidal Figures of Efquilibrium (New York: Dover)

Chandrasekhar, S., \& Elbert, D. D. 1974, Astrophys. J., 192, 731, erratum: 1978, 220, 303

Chandrasekhar, S., \& Nutku, Y. 1969, Astrophys. J., 158,55

Ferrers, N. M. 1875, Quarterly Journal of Pure and Applied Mathematics, 14, 1

Friedman, J., \& Stergioulas, N. 2013, Rotating Relativistic Stars (Cambridge: Cambridge Univ. Press)

Geroch, R. 1971, J. Math. Phys., 12, 918
Griffiths, J. B., \& Podolsky, J. 2009, Exact spacetimes in Einstein's general relativity (Cambridge: Cambridge University Press)

Gürlebeck, N., \& Petroff, D. 2010, Astrophys. J., 722,1207

Hagihara, Y. 1970, Theories of equilibrium figures of a rotating homogeneous fluid mass. (Washington D.C.: NASA Special Publication)

Israel, W. 1977, Phys. Rev. D, 15, 935

Lindblom, L. 1976, Astrophys. J., 208, 873

Lindblom, L. 1992, Phil. Trans. R. Soc. Lond. A, 340,353

Meinel, R., Ansorg, M., Kleinwächter, A., Neugebauer, G., \& Petroff, D. 2008, Relativistic Figures of Equilibrium (Cambridge: Cambridge University Press)

Petroff, D. 2003a, PhD thesis, Friedrich-SchillerUniversität, Jena

—. 2003b, Phys. Rev. D, 68, 104029

Taniguchi, K., Asada, H., \& Shibata, M. 1998, Prog. Theor. Phys., 100, 703

This 2-column preprint was prepared with the AAS LATEX macros v5.2. 


\section{A. Higher moments}

The interior solutions for the higher moments of the homogeneous mass distribution were obtained in Ferrers (1877). They are given in Chandrasekhar (1987) and we repeat them here:

$$
\begin{aligned}
D_{i} & =\pi G \mu a_{i}^{2} x^{i}\left(A_{i}-\sum_{l=1}^{3} A_{i l}\left(x^{l}\right)^{2}\right) \\
D_{i j} & =\pi G \mu a_{i}^{2}\left(a_{j}^{2}\left(A_{i j}-\sum_{l=1}^{3} A_{i j l}\left(x^{l}\right)^{2}\right) x^{i} x^{j}+\frac{1}{4} \delta_{i j}\left(B_{i}-2 \sum_{l=1}^{3} B_{i l}\left(x^{l}\right)^{2}+\sum_{l=1}^{3} \sum_{k=1}^{3} B_{i l k}\left(x^{l}\right)^{2}\left(x^{k}\right)^{2}\right)\right) \\
D_{i j k} & =\pi G \mu a_{i}^{2} a_{j}^{2} a_{k}^{2}\left(A_{i j k}-\sum_{l=1}^{3} A_{i j k l}\left(x^{l}\right)^{2}\right) x^{i} x^{j} x^{k}+\frac{1}{4}\left(V_{i j k}+V_{j k i}+V_{k i j}\right) \\
V_{i j k} & =a_{i}^{2} a_{j}^{2} \delta_{j k}\left(B_{i j}-\sum_{l=1}^{3}\left(2 B_{i j l}-\sum_{m=1}^{3} B_{i j l m}\left(x^{m}\right)^{2}\right)\left(x^{l}\right)^{2}\right) x^{i} .
\end{aligned}
$$

\section{B. Explicit analytical results}

We present the results that are too lengthy for the main text.

\section{B.1. The angular momentum}

In Section 3.4.1, we discussed the $1 \mathrm{PN}$ contribution to the angular momentum. We gave the analytic expression for the Newtonian angular momentum in Equation (35) and the plot for the $1 \mathrm{PN}$ contribution, cf. Figure 1(b), Here, we provide the analytic expression for $L^{(2)}$, too:

$$
\begin{aligned}
L^{(2)}= & L^{(0)} \frac{\pi G}{7} \mu\left(-26 a_{1}^{2} A_{1}-\frac{1}{\left(a_{1}^{2}-a_{2}^{2}\right)}\left(\left(5 a_{1}^{2}+19 a_{2}^{2}\right) a_{1}^{2} S_{1}+\left(19 a_{1}^{2}+5 a_{2}^{2}\right) a_{1}^{2} S_{2}+4\left(a_{1}^{2}-3 a_{2}^{2}\right) \times\right.\right. \\
& \left.a_{1}^{2} S_{3}+a_{2}^{2}\left(7 a_{1}^{2}+a_{2}^{2}\right) S_{4}\right)-a_{3}^{2} S_{5}+2\left(3 a_{1}^{4} A_{11}+2 a_{1}^{2}\left(a_{2}^{2}\left(6\left(B_{112}+B_{122}\right)+A_{12}+3 B_{123}\right)+\right.\right. \\
& \left.4 B_{12}+3 B_{23}\right)+a_{2}^{2}\left(8 B_{12}+3 a_{2}^{2}\left(2 B_{123}+A_{22}\right)+6 B_{23}\right)+a_{3}^{2}\left(a_{1}^{2} A_{13}+a_{2}^{2} A_{23}-2 A_{3}\right)+ \\
& \left.\left.21 A_{\emptyset}\right)-26 a_{2}^{2} A_{2}\right)-\frac{4 \pi^{5 / 2} a_{3} a_{1}^{3} a_{2}^{3} \mu(G \mu)^{3 / 2}}{105\left(a_{1}^{2}-a_{2}^{2}\right)}\left(\left(19 a_{1}^{2}+5 a_{2}^{2}\right) w_{1}-\left(5 a_{1}^{2}+19 a_{2}^{2}\right) w_{2}\right) .
\end{aligned}
$$

\section{B.2. The surface condition}

Our numerical results and the numerical results in Paper II do not coincide as discussed in detail in Gürlebeck \& Petroff (2010). We believe that there is a problem in the numerical evaluation of the right hand side of Equation (33) in Paper II. However, this could not be explicitly seen since those expressions were not given. We provide the lengthy analytical expression for Equation (33), which we obtained and used in all numerical considerations in our text. To shorten the results, we introduce a third kind of index symbols

$$
C_{i_{1} \ldots i_{k}}=\int_{0}^{\infty} \frac{u^{2}}{H(u) z_{i_{1}} z_{i_{2}} \cdots z_{i_{k}}} d u, \quad C_{i_{1} \ldots i_{k}}\left(a_{1}, a_{2}, a_{3}\right)=a_{1}^{6-2 k} C_{i_{1} \ldots i_{k}}\left(1, \bar{a}_{2}, \bar{a}_{3}\right)=a_{1}^{6-2 k} \bar{C}_{i_{1} \ldots i_{k}}
$$


with the same meaning of $H(u)$ and $z_{i}$ as in Equation (4). The coefficient matrix $\left(M_{i j}\right)$ reads

$$
\begin{aligned}
& M_{00}=\frac{1}{\pi^{2}} \\
& M_{01}=3 \bar{a}_{3}^{2} \bar{B}_{33}-\bar{B}_{13} \\
& M_{02}=3 \bar{a}_{3}^{2} \bar{B}_{33}-\bar{a}_{2}^{2} \bar{B}_{23} \\
& M_{03}=\frac{1}{4}\left(\bar{a}_{2}^{2}-1\right)\left(\bar{C}_{112}-2 \bar{a}_{3}^{2} \bar{C}_{1123}+\bar{a}_{3}^{4} \bar{C}_{11233}\right) \\
& M_{04}=-\frac{\bar{a}_{2}^{2}}{4}\left(\left(\bar{a}_{2}^{2}-\bar{a}_{3}^{2}\right)\left(\bar{C}_{223}-2 \bar{a}_{3}^{2} \bar{C}_{2233}+\bar{a}_{3}^{4} \bar{C}_{22333}\right)+4 \bar{a}_{3}^{4} \bar{C}_{2333}\right) \\
& M_{05}=-\frac{\bar{a}_{3}^{2}}{12}\left(\bar{a}_{3}^{2}\left(11 \bar{C}_{133}+\bar{a}_{3}^{2}\left(-10 \bar{C}_{1333}+11\left(\bar{a}_{3}^{2}-1\right) \bar{C}_{13333}+24 \bar{a}_{3}^{2} \bar{B}_{3333}\right)+14 \bar{C}_{1333}\right)-3 \bar{C}_{133}\right) \\
& M_{10}=M_{20}=M_{30}=M_{40}=M_{50}=0 \\
& M_{11}=\frac{2}{\bar{a}_{2}^{2}}\left(\left(\bar{a}_{3}^{2}-1\right)\left(\bar{a}_{3}^{2}-\bar{a}_{2}^{2}\right) \bar{C}_{1233}-2 \bar{a}_{3}^{2} \bar{B}_{33}\right) \\
& M_{12}=\frac{2}{\bar{a}_{2}^{2}}\left(3\left(\bar{a}_{2}^{2}-\bar{a}_{3}^{2}\right)^{2} \bar{C}_{2233}-4 \bar{a}_{3}^{2} \bar{B}_{23}\right) \\
& M_{13}=\frac{1}{\bar{a}_{2}^{2}}\left(\left(\left(\bar{a}_{2}^{2}-1\right) \bar{a}_{3}^{2}+\bar{a}_{2}^{2}\right) \bar{C}_{1123}-\left(\bar{a}_{2}^{2}-1\right) \bar{a}_{3}^{4} \bar{C}_{11233}-3 \bar{a}_{2}^{4} \bar{C}_{1223}\right) \\
& M_{14}=5 \bar{a}_{2}^{4} \bar{C}_{2223}-4 \bar{a}_{3}^{4} \bar{a}_{2}^{4} \bar{A}_{2233}-2\left(\bar{a}_{2}^{4}+\bar{a}_{3}^{2} \bar{a}_{2}^{2}+\bar{a}_{3}^{4}\right) \bar{C}_{2233}+5 \bar{a}_{3}^{4} \bar{C}_{2333}+4 \bar{a}_{3}^{2} \bar{A}_{3} \\
& M_{15}=\frac{\bar{a}_{3}^{2}}{3 \bar{a}_{2}^{2}}\left(3 \bar{a}_{3}^{4}\left(-2 \bar{C}_{1233}+3 \bar{C}_{1333}+5 \bar{C}_{2333}-9 \bar{C}_{3333}\right)+\bar{a}_{3}^{2}\left(3\left(\bar{a}_{2}^{2}-1\right) \bar{C}_{1233}+6 \bar{C}_{1333}+8 \bar{A}_{3}\right)-\right. \\
& \left.3 \bar{a}_{2}^{2} \bar{C}_{1233}-8 \bar{a}_{3}^{8} \bar{A}_{3333}\right) \\
& M_{21}=-M_{22}=\frac{48\left(2 \bar{a}_{2}^{2}+1\right) \bar{B}_{12}}{\bar{a}_{2}^{2}\left(\bar{a}_{2}^{2}-1\right)} \\
& M_{23}=\left(\left(\frac{12}{\bar{a}_{2}^{2}}-6\right) \bar{a}_{3}^{2}-6\right) \bar{C}_{11223}+\frac{6\left(\bar{a}_{2}^{2}-1\right) \bar{a}_{3}^{4} \bar{C}_{11233}}{\bar{a}_{2}^{4}}+\frac{\left(48-96 \bar{a}_{2}^{2}\right) \bar{B}_{12}}{\bar{a}_{2}^{2}-\bar{a}_{2}^{4}}+30\left(\bar{a}_{2}^{2}-\bar{a}_{3}^{2}\right) \bar{C}_{12223} \\
& M_{24}=-\frac{16\left(\bar{a}_{2}^{2}+2\right) \bar{B}_{12}}{\bar{a}_{2}^{2}-1}+16 \bar{a}_{2}^{6} \bar{A}_{2222}+54\left(\bar{a}_{3}^{2}-\bar{a}_{2}^{2}\right) \bar{a}_{2}^{2} \bar{C}_{22223}+12\left(2 \bar{a}_{2}^{4}-\bar{a}_{3}^{2} \bar{a}_{2}^{2}-\bar{a}_{3}^{4}\right) \bar{C}_{22233}+ \\
& 48 \bar{a}_{3}^{4} \bar{a}_{2}^{2} \bar{A}_{2233}+30 \bar{a}_{3}^{4}\left(\frac{\bar{a}_{3}^{2}}{\bar{a}_{2}^{2}}-1\right) \bar{C}_{22333}-\frac{64 \bar{a}_{3}^{2} \bar{A}_{3}}{\bar{a}_{2}^{2}} \\
& M_{25}=\frac{2}{\bar{a}_{2}^{4}}\left(-3 \bar{a}_{2}^{4}\left(\bar{a}_{3}^{2}-1\right) \bar{a}_{3}^{2} \bar{C}_{12233}+3\left(4 \bar{a}_{3}^{4}+\bar{a}_{3}^{2}-\bar{a}_{2}^{2}\left(3 \bar{a}_{3}^{2}+2\right)\right) \bar{a}_{3}^{4} \bar{C}_{12333}+\right. \\
& \left.27\left(\bar{a}_{2}^{2}-\bar{a}_{3}^{2}\right) \bar{a}_{3}^{6} \bar{C}_{23333}-8 \bar{a}_{3}^{4} \bar{A}_{3}+8 \bar{a}_{3}^{10} \bar{A}_{3333}\right) \\
& M_{31}=-2\left(\left(\bar{a}_{3}^{2}-1\right)\left(3 \bar{B}_{113}+\bar{a}_{3}^{2} \bar{B}_{133}\right)+4 \bar{a}_{3}^{2} \bar{B}_{33}\right) \\
& M_{32}=2\left(\left(\bar{a}_{3}^{2}-1\right)\left(\bar{a}_{3}^{2}-\bar{a}_{2}^{2}\right) \bar{C}_{1233}-2 \bar{a}_{3}^{2} \bar{B}_{33}\right) \\
& M_{33}=-5\left(\bar{a}_{2}^{2}-1\right) \bar{C}_{1112}+5\left(\bar{a}_{2}^{2}-1\right) \bar{a}_{3}^{2} \bar{C}_{11123}+\left(\left(\bar{a}_{3}^{2}+4\right) \bar{a}_{2}^{2}-\bar{a}_{3}^{2}-2\right) \bar{C}_{1123}-\left(\bar{a}_{2}^{2}-1\right) \bar{a}_{3}^{4} \bar{C}_{11233} \\
& M_{34}=\left(\bar{a}_{2}^{4}+\left(\bar{a}_{2}^{2}-1\right) \bar{a}_{3}^{2} \bar{a}_{2}^{2}\right) \bar{C}_{1223}-3 \bar{a}_{2}^{2} \bar{a}_{3}^{4} \bar{C}_{1233}+\bar{a}_{2}^{2}\left(\bar{a}_{3}^{2}-2 \bar{a}_{2}^{2}\right) \bar{a}_{3}^{2} \bar{C}_{2233}+5 \bar{a}_{2}^{2} \bar{a}_{3}^{4} \bar{C}_{2333} \\
& M_{35}=-3 \bar{a}_{3}^{2} \bar{B}_{113}+3\left(-2 \bar{a}_{3}^{4}+\bar{a}_{3}^{2}+1\right) \bar{a}_{3}^{4} \bar{C}_{11333}+2\left(\bar{a}_{3}^{2}+1\right) \bar{a}_{3}^{4} \bar{B}_{133}+\left(6 \bar{a}_{3}^{2}-7\right) \bar{a}_{3}^{6} \bar{B}_{1333}+\frac{20}{3} \bar{a}_{3}^{4} \bar{A}_{3}- \\
& \frac{20}{3} \bar{a}_{3}^{8} \bar{A}_{333}-5 \bar{a}_{3}^{6} \bar{B}_{333}+\frac{35}{3} \bar{a}_{3}^{8} \bar{B}_{3333}
\end{aligned}
$$




$$
\begin{aligned}
M_{41}= & -M_{42}=\frac{48 \bar{B}_{12}}{\bar{a}_{2}^{2}-1} \\
M_{43}= & 10\left(\bar{a}_{2}^{2}-1\right) \bar{C}_{11122}-\frac{10\left(\bar{a}_{2}^{2}-1\right) \bar{a}_{3}^{2} \bar{C}_{11123}}{\bar{a}_{2}^{2}}-8 \bar{a}_{2}^{2} \bar{A}_{1122}-\frac{2\left(\bar{a}_{2}^{2}-1\right)\left(\bar{a}_{2}^{2}\left(3 \bar{a}_{3}^{2}+2\right)-2 \bar{a}_{3}^{2}\right) \bar{C}_{11223}}{\bar{a}_{2}^{2}}+ \\
& \frac{2\left(\bar{a}_{2}^{2}-1\right) \bar{a}_{3}^{4} \bar{C}_{11233}}{\bar{a}_{2}^{2}}+\frac{16 \bar{B}_{12}}{\bar{a}_{2}^{2}-1}+\frac{8 \bar{a}_{3}^{2} \bar{A}_{3}}{\bar{a}_{2}^{2}} \\
M_{44}= & -\frac{16 \bar{a}_{2}^{2} \bar{B}_{12}}{\bar{a}_{2}^{2}-1}+\left(2\left(3 \bar{a}_{3}^{2}-5\right) \bar{a}_{2}^{4}+4 \bar{a}_{3}^{2} \bar{a}_{2}^{2}\right) \bar{C}_{12223}-4\left(\bar{a}_{3}^{2}-1\right) \bar{a}_{2}^{4} \bar{C}_{12233}+6 \bar{a}_{3}^{4}\left(\bar{a}_{3}^{2}-1\right) \bar{C}_{12333}+ \\
& 4 \bar{a}_{3}^{2}\left(\bar{a}_{2}^{2}-\bar{a}_{3}^{2}\right) \bar{a}_{2}^{2} \bar{C}_{22233}+8 \bar{a}_{3}^{4} \bar{a}_{2}^{4} \bar{A}_{2233}+4 \bar{a}_{3}^{4}\left(\bar{a}_{3}^{2}-\bar{a}_{2}^{2}\right) \bar{C}_{22333}-8 \bar{a}_{3}^{2} \bar{A}_{3} \\
M_{45}= & 6 \bar{a}_{3}^{2} \bar{B}_{1123}-\frac{12\left(\bar{a}_{3}^{2}-1\right) \bar{a}_{3}^{4} \bar{B}_{1133}}{\bar{a}_{2}^{2}}+\frac{18\left(\bar{a}_{3}^{2}-1\right) \bar{a}_{3}^{4} \bar{C}_{11333}}{\bar{a}_{2}^{2}}-4\left(\bar{a}_{3}^{6}+\bar{a}_{3}^{4}\right) \bar{B}_{1233}+\frac{8 \bar{a}_{3}^{8} \bar{A}_{1333}}{\bar{a}_{2}^{2}}+ \\
& \frac{2\left(4 \bar{a}_{3}^{8}+\bar{a}_{3}^{6}\right) \bar{B}_{1333}}{\bar{a}_{2}^{2}}+10 \bar{a}_{3}^{6} \bar{B}_{2333}-\frac{40 \bar{a}_{3}^{4} \bar{A}_{3}}{3 \bar{a}_{2}^{2}}+\frac{16 \bar{a}_{3}^{10} \bar{A}_{3333}}{3 \bar{a}_{2}^{2}}-\frac{18 \bar{a}_{3}^{8} \bar{B}_{3333}}{\bar{a}_{2}^{2}} \\
M_{51}= & -M_{52}=\frac{48\left(\bar{a}_{2}^{2}+2\right) \bar{B}_{12}}{\bar{a}_{2}^{2}-1} \\
M_{53}= & 16 \bar{A}_{1111}+54\left(\bar{a}_{2}^{2}-1\right) \bar{C}_{1112}-12\left(\left(\bar{a}_{3}^{2}+4\right) \bar{a}_{2}^{2}-3 \bar{a}_{3}^{2}-2\right) \bar{C}_{11123}+6\left(\bar{a}_{2}^{2}-1\right) \bar{a}_{3}^{4} \bar{C}_{11233}+ \\
& \frac{16\left(5 \bar{a}_{2}^{2}-2\right) \bar{B}_{12}}{\bar{a}_{2}^{2}-1}=16 \bar{a}_{3}^{2} \bar{A}_{3} \\
M_{54}= & \left(6 \bar{a}_{2}^{2} \bar{a}_{3}^{2}-6 \bar{a}_{2}^{4}\right) \bar{C}_{11223}-\frac{48 \bar{a}_{2}^{2} \bar{B}_{12}}{\bar{a}_{2}^{2}-1}+12 \bar{a}_{2}^{2}\left(\bar{a}_{2}^{2}-\bar{a}_{3}^{2}\right) \bar{a}_{3}^{2} \bar{C}_{12233}+24 \bar{a}_{2}^{2}\left(\bar{a}_{3}^{2}-1\right) \bar{a}_{3}^{4} \bar{C}_{12333}+ \\
& \left(6 \bar{a}_{2}^{2} \bar{a}_{3}^{6}-6 \bar{a}_{2}^{4} \bar{a}_{3}^{4}\right) \bar{C}_{22333} \\
M_{55}= & -30\left(\bar{a}_{3}^{2}-1\right) \bar{a}_{3}^{2} \bar{C}_{11133}+12\left(6 \bar{a}_{3}^{4}-5 \bar{a}_{3}^{2}-1\right) \bar{a}_{3}^{4} \bar{C}_{11333}+48 \bar{a}_{3}^{8} \bar{A}_{1333}-48\left(\bar{a}_{3}^{2}-1\right) \bar{a}_{3}^{6} \bar{B}_{1333}- \\
& 54\left(\bar{a}_{3}^{2}-1\right) \bar{a}_{3}^{6} \bar{C}_{13333}-64 \bar{a}_{3}^{4} \bar{A}_{3}+16 \bar{a}_{3}^{10} \bar{A}_{3333} .
\end{aligned}
$$

The inhomogeneities are given by

$$
\begin{aligned}
& b_{0}=-\frac{1}{4}\left(-2 \bar{C}_{13}\left(\bar{A}_{1}-2 \bar{a}_{2}^{2} \bar{B}_{12}\right)-\bar{a}_{3}^{2}\left(-2 \bar{A}_{1}\left(\bar{C}_{133}+\bar{C}_{233}\right)+\bar{A}_{3}\left(3\left(\bar{B}_{1}+\bar{B}_{2}\right)+\bar{C}_{33}\right)+\right.\right. \\
& \left.2 \bar{B}_{12}\left(\bar{a}_{2}^{2}\left(2 \bar{C}_{133}-\bar{C}_{233}\right)+3 \bar{C}_{233}\right)\right)-2 \bar{C}_{23}\left(\bar{A}_{1}+\left(\bar{a}_{2}^{2}-3\right) \bar{B}_{12}\right)+\bar{a}_{3}^{6} \bar{A}_{3}\left(3\left(\bar{B}_{133}+\bar{B}_{233}\right)-16 \bar{B}_{333}\right)+ \\
& \left.\bar{a}_{3}^{4} \bar{A}_{3}\left(6\left(\bar{C}_{133}+\bar{C}_{233}\right)-8 \bar{A}_{3}+9 \bar{C}_{333}\right)+8 \bar{A}_{\emptyset}^{2}\right) \\
& b_{1}=-\frac{1}{a_{2}^{2}}\left(\bar{A}_{1}\left(2 \bar{a}_{2}^{2} \bar{C}_{123}-2 \bar{a}_{3}^{2} \bar{C}_{133}\right)+\bar{B}_{12}\left(-8\left(\bar{a}_{2}^{2}-1\right) \bar{a}_{3}^{2} \bar{B}_{123}-4 \bar{a}_{2}^{4} \bar{C}_{123}-4\left(\bar{a}_{2}^{2}-2\right) \bar{a}_{3}^{2} \bar{B}_{13}-4 \bar{a}_{3}^{4} \bar{a}_{2}^{2} \bar{B}_{133}+\right.\right. \\
& \left.8 \bar{a}_{2}^{4} \bar{B}_{223}-4 \bar{a}_{2}^{2} \bar{C}_{223}+16 \bar{a}_{3}^{2} \bar{a}_{2}^{2} \bar{A}_{23}+4 \bar{a}_{3}^{2} \bar{C}_{233}+8\left(\bar{a}_{2}^{2}-\bar{a}_{3}^{2}\right) \bar{A}_{3}-16 \bar{a}_{2}^{2}\right)+\bar{A}_{\emptyset}\left(8 \bar{a}_{2}^{2} \bar{B}_{12}+4\left(\bar{a}_{3}^{2}-\bar{a}_{2}^{2}\right) \bar{B}_{23}\right)+ \\
& \bar{A}_{3}\left(3 \bar{a}_{2}^{2} \bar{a}_{3}^{2} \bar{C}_{123}-3 \bar{a}_{3}^{4} \bar{C}_{133}-6 \bar{a}_{2}^{4} \bar{a}_{3}^{2} \bar{B}_{223}+3 \bar{a}_{2}^{2} \bar{a}_{3}^{2} \bar{C}_{223}+6\left(\bar{a}_{3}^{2}-\bar{a}_{2}^{2}\right) \bar{a}_{3}^{2} \bar{B}_{23}+\left(5 \bar{a}_{2}^{2} \bar{a}_{3}^{2}-3 \bar{a}_{3}^{4}\right) \bar{C}_{233}+\right. \\
& \left.\left.10\left(\bar{a}_{2}^{2}-\bar{a}_{3}^{2}\right) \bar{a}_{3}^{6} \bar{B}_{2333}+10 \bar{a}_{3}^{6} \bar{B}_{333}-5 \bar{a}_{3}^{4} \bar{C}_{333}\right)+\bar{A}_{2}\left(-4 \bar{a}_{2}^{6} \bar{B}_{223}+2 \bar{a}_{2}^{4} \bar{C}_{223}-2 \bar{a}_{3}^{2} \bar{a}_{2}^{2} \bar{C}_{233}\right)+\left(w_{2}-w_{1}\right) \bar{a}_{2}^{3} \bar{\Omega}\right) \\
& b_{2}=-\frac{6}{a_{2}^{4}}\left(2 \bar{A}_{1}\left(\bar{a}_{2}^{2}\left(4 \bar{B}_{12}+\bar{a}_{3}^{2}\left(\bar{C}_{1223}+\bar{C}_{1233}\right)\right)-\bar{a}_{2}^{4} \bar{C}_{1223}-\bar{a}_{3}^{4} \bar{C}_{1233}\right)+4 \bar{B}_{12}\left(\overline { a } _ { 2 } ^ { 4 } \left(6 \bar{B}_{112}+6 \bar{B}_{122}-\right.\right.\right. \\
& \left.2 \bar{a}_{3}^{2}\left(\bar{B}_{123}+2 \bar{A}_{223}\right)+2 \bar{B}_{123}+7 \bar{B}_{222}+4 \bar{B}_{23}\right)+\bar{a}_{2}^{6}\left(\bar{B}_{122}+4 \bar{B}_{123}+2 \bar{A}_{222}\right)+\bar{a}_{2}^{2}\left(2 \overline { a } _ { 3 } ^ { 2 } \left(2\left(\bar{B}_{123}+\bar{B}_{13}\right)-\right.\right. \\
& \left.\left.\left.5 \bar{B}_{223}\right)+\bar{a}_{3}^{4} \bar{B}_{133}+2 \bar{B}_{23}\right)+\bar{a}_{3}^{2}\left(-4\left(\bar{B}_{123}+\bar{B}_{13}\right)+\bar{a}_{3}^{2} \bar{B}_{233}+4 \bar{A}_{3}\right)\right)+2 \bar{a}_{2}^{2} \bar{A}_{2}\left(( \overline { a } _ { 2 } ^ { 2 } - \overline { a } _ { 3 } ^ { 2 } ) \left(4 \bar{a}_{2}^{4} \bar{B}_{2223}-\right.\right. \\
& \left.\left.\bar{a}_{2}^{2} \bar{C}_{2223}+\bar{a}_{3}^{2} \bar{C}_{2233}\right)-4\left(\bar{a}_{2}^{2}+1\right) \bar{B}_{12}\right)+20 \bar{a}_{2}^{4} \bar{B}_{12}^{2}+\bar{a}_{3}^{2}\left(\bar{a}_{3}^{2}-\bar{a}_{2}^{2}\right) \bar{A}_{3}\left(\bar{a}_{2}^{2}\left(3\left(\bar{C}_{1223}+\bar{C}_{2223}\right)+5 \bar{C}_{2233}\right)+\right. \\
& \left.\left.\bar{a}_{3}^{2}\left(-3\left(\bar{C}_{1233}+\bar{C}_{2233}\right)+20 \bar{a}_{3}^{2} \bar{B}_{2333}-5 \bar{C}_{2333}\right)-12 \bar{a}_{2}^{4} \bar{B}_{2223}\right)+\frac{2\left(w_{1}+w_{2}\right)\left(2 \bar{a}_{2}^{2}+1\right) \bar{a}_{2}^{3} \bar{\Omega}}{\bar{a}_{2}^{2}-1}\right)
\end{aligned}
$$




$$
\begin{aligned}
& b_{3}=-\left(-2 \bar{A}_{1}\left(2 \bar{B}_{113}-\bar{C}_{113}+4 \bar{a}_{2}^{2} \bar{B}_{12}+\bar{a}_{3}^{2}\left(\bar{C}_{133}+3 \bar{A}_{3}\right)\right)+4 \bar{A}_{\emptyset}\left(-\bar{A}_{1}+2 \bar{B}_{12}+\bar{a}_{3}^{2} \bar{A}_{3}\right)+3 \bar{a}_{3}^{2} \bar{B}_{11} \bar{A}_{3}+\right. \\
& \bar{B}_{12}\left(\bar{a}_{3}^{2}\left(-4 \bar{B}_{123}-8 \bar{B}_{13}+4 \bar{C}_{233}+3 \bar{A}_{3}\right)-4 \bar{a}_{2}^{2}\left(-2 \bar{B}_{113}+\bar{C}_{113}-\bar{a}_{3}^{2}\left(2 \bar{B}_{123}+4 \bar{A}_{13}+3 \bar{B}_{13}-2 \bar{A}_{3}\right)+\right.\right. \\
& \left.\left.\bar{a}_{3}^{4} \bar{B}_{133}\right)\right)+\bar{a}_{3}^{2} \bar{A}_{3}\left(-3\left(\bar{a}_{3}^{2}+2\right) \bar{B}_{113}+\bar{a}_{3}^{2}\left(-\left(3 \bar{B}_{123}+3 \bar{C}_{133}+5 \bar{a}_{3}^{2}\left(2\left(\bar{a}_{3}^{2}-1\right) \bar{B}_{1333}-\bar{C}_{1333}-2 \bar{B}_{333}\right)+\right.\right.\right. \\
& \left.\left.\left.\left.5 \bar{C}_{1333}+3 \bar{C}_{233}+5 \bar{B}_{33}\right)\right)+5 \bar{B}_{13}\right)-2 \bar{a}_{2}^{2} \bar{A}_{2}\left(3 \bar{B}_{12}+\bar{a}_{3}^{2}\left(\bar{B}_{123}+\bar{C}_{233}\right)\right)-4 \bar{B}_{12}^{2}+6 \bar{a}_{3}^{4} \bar{A}_{3}^{2}+\left(w_{2}-w_{1}\right) \bar{a}_{2} \bar{\Omega}\right) \\
& b_{4}=-\frac{2}{a_{2}^{2}}\left(2\left(\bar{a}_{2}^{2}-\bar{a}_{3}^{2}\right) \bar{A}_{1}\left(2 \bar{B}_{1123}-\bar{C}_{1123}+\bar{a}_{3}^{2} \bar{C}_{1233}\right)+4 \bar{B}_{12}\left(\overline { a } _ { 2 } ^ { 2 } \left(6 \bar{B}_{112}+\bar{a}_{3}^{2}\left(-3\left(\bar{C}_{1123}+\bar{C}_{1223}\right)+\right.\right.\right.\right. \\
& \left.\left.\bar{a}_{3}^{2} \bar{C}_{1233}+2 \bar{B}_{13}\right)+3\left(\bar{C}_{1223}+2 \bar{B}_{23}\right)\right)+\bar{a}_{2}^{4}\left(3 \bar{C}_{1123}+6 \bar{B}_{122}+6 \bar{B}_{123}-\bar{a}_{3}^{2} \bar{C}_{1233}\right)+ \\
& \left.\bar{a}_{3}^{2}\left(\left(\bar{a}_{3}^{2}-1\right) \bar{C}_{1233}+2 \bar{B}_{23}\right)\right)+\bar{a}_{3}^{2} \bar{A}_{3}\left(\overline { a } _ { 3 } ^ { 2 } \left(3\left(\bar{C}_{1123}+\bar{C}_{1223}\right)-6 \bar{B}_{1123}-\bar{a}_{3}^{2}\left(3\left(\bar{C}_{1233}+\bar{C}_{2233}\right)+10 \bar{B}_{1333}+\right.\right.\right. \\
& \left.\left.5 \bar{C}_{2333}\right)+5 \bar{C}_{1233}+10 \bar{a}_{3}^{4}\left(\bar{B}_{1333}+\bar{B}_{2333}\right)\right)+\bar{a}_{2}^{2}\left(-3\left(\bar{C}_{1123}+\bar{C}_{1223}\right)+6 \bar{B}_{1123}+\bar{a}_{3}^{2}\left(3\left(\bar{C}_{1233}+\bar{C}_{2233}\right)-\right.\right. \\
& \left.\left.\left.10 \bar{a}_{3}^{2} \bar{B}_{2333}+5 \bar{C}_{2333}\right)-5 \bar{C}_{1233}\right)-6\left(\bar{a}_{3}^{2}-1\right) \bar{a}_{2}^{4} \bar{B}_{1223}\right)+12 \bar{a}_{2}^{2} \bar{B}_{12}^{2}+2 \bar{a}_{2}^{2} \bar{A}_{2}\left(-2\left(\bar{a}_{3}^{2}-1\right) \bar{a}_{2}^{4} \bar{B}_{1223}+\right. \\
& \left.\left.\left(\bar{a}_{3}^{2}-\bar{a}_{2}^{2}\right) \bar{C}_{1223}+\bar{a}_{3}^{2}\left(\bar{a}_{2}^{2}-\bar{a}_{3}^{2}\right) \bar{C}_{2233}\right)+\frac{6\left(w_{1}+w_{2}\right) \bar{a}_{2}^{3} \bar{\Omega}}{\bar{a}_{2}^{2}-1}\right) \\
& b_{5}=-6\left(2\left(\bar{a}_{3}^{2}-1\right) \bar{A}_{1}\left(-5 \bar{B}_{1113}+\bar{B}_{113}-\bar{a}_{3}^{2} \bar{C}_{1133}\right)-24 \bar{a}_{2}^{2} \bar{C}_{111} \bar{B}_{12}-20 \bar{a}_{2}^{2} \bar{B}_{111} \bar{B}_{12}+\right. \\
& 4 \bar{B}_{12}\left(\bar{a}_{2}^{2}\left(4\left(\bar{a}_{3}^{2}-1\right) \bar{B}_{1113}+6 \bar{B}_{112}-2 \bar{a}_{3}^{2} \bar{B}_{113}+6 \bar{B}_{122}+4 \bar{B}_{123}+4 \bar{B}_{13}+\bar{a}_{3}^{4} \bar{B}_{133}+2 \bar{B}_{23}\right)+\bar{B}_{112}+\right. \\
& \left.2 \bar{a}_{3}^{2}\left(\bar{B}_{123}+2 \bar{C}_{123}\right)+2 \bar{a}_{2}^{4} \bar{B}_{123}+4 \bar{B}_{23}+\bar{a}_{3}^{4} \bar{B}_{233}\right)+\bar{a}_{3}^{2}\left(\bar{a}_{3}^{2}-1\right) \bar{A}_{3}\left(3\left(\bar{C}_{1113}+\bar{C}_{1123}\right)-12 \bar{B}_{1113}-\right. \\
& \left.\bar{a}_{3}^{2}\left(3\left(\bar{C}_{1133}+\bar{C}_{1233}\right)+5 \bar{C}_{1333}\right)+5 \bar{C}_{1133}+20 \bar{a}_{3}^{4} \bar{B}_{1333}\right)+2\left(\bar{a}_{3}^{2}-1\right) \bar{a}_{2}^{2} \bar{A}_{2}\left(\bar{C}_{1123}-\bar{a}_{3}^{2} \bar{C}_{1233}\right)+ \\
& \left.4\left(2 \bar{a}_{2}^{2}+3\right) \bar{B}_{12}^{2}+\frac{2\left(w_{1}+w_{2}\right)\left(\bar{a}_{2}^{2}+2\right) \bar{a}_{2} \bar{\Omega}}{\bar{a}_{2}^{2}-1}\right) \text {. }
\end{aligned}
$$

\section{The limit $\bar{a}_{3} \rightarrow 0$ to $1 \mathrm{PN}$ order}

In Gürlebeck \& Petroff (2010), we took a close look at the axisymmetric limit $\left(\bar{a}_{2} \rightarrow 1\right)$ of the PN Dedekind ellipsoids. It turns out that the PN Maclaurin spheroids emerge in the limit but only if the PN velocity field is generalized as in Equation (25). Here we consider the opposite limit of a rod along the $x_{1}$-axis, i.e., $\bar{a}_{2} \rightarrow 0$, cf. Section 2.1. We begin by deriving conditions that arise from the behavior of the metric functions in this limit, where it will be important to treat $\mu$ and $a_{1}$ as functions of $\bar{a}_{2}$. We then examine the surface and conclude that the only acceptable solution is a member of the Weyl class, i.e., an axially symmetric and static spacetime. The matter content collapses to a singularity along the axis and the limiting spacetimes contain the Levi-Civita spacetime and the Curzon-Chazy particle in the special cases in which the rod has infinite length $\left(a_{1} \rightarrow \infty\right)$ or zero length $\left(a_{1} \rightarrow 0\right)$, see e.g. Griffiths \& Podolsky (2009). In order to include the Levi-Civita metric in the subsequent derivations, we shall divide up the ellipsoid into slices defined by $x^{1}=x_{0}^{1}$ and with a thickness $\delta x^{1}$, which we denote by $\mathcal{S}\left(\delta x^{1}, x_{0}^{1}\right)$.

Let us start by looking at $\Phi$, which is determined by the Poisson equation (24a). The inhomogeneity is a sum of (Newtonian) kinetic, inner and potential energy densities. Beginning with the kinetic energy contained in $\mathcal{S}$, we find

$$
\int_{\mathcal{S}} \frac{\mu}{2} \mathbf{v}^{2} d^{3} x \propto a_{1}^{4} \bar{a}_{2} \bar{a}_{3} \delta x^{1} \mu^{2} \bar{B}_{12}\left(1-\left(\frac{x_{0}^{1}}{a_{1}}\right)^{2}\right)\left(1+\left(4 \bar{a}_{2}^{2}-1\right)\left(\frac{x_{0}^{1}}{a_{1}}\right)^{2}+O\left(\frac{\delta x^{1}}{a_{1}}\right)\right) .
$$

It turns out that the ratio of the inner to the kinetic energy tends to zero in the limit. Furthermore, the potential energy is proportional to the kinetic one. Thus, it suffices to derive the form of the kinetic energy density in the limit. We choose it to be a line energy density as required for a well-defined Equation (24a). 
This then implies

$$
\mu=\frac{e_{\max }^{\frac{1}{2}}}{\pi \sqrt{G} a_{1}^{2} \bar{a}_{3}^{2}\left(-\ln \bar{a}_{3}\right)^{\frac{1}{2}}}
$$

whereby $e_{\max }$ is an arbitrary constant and the logarithmic term comes from the expansion of $\bar{B}_{12}$. This yields a source for the potential $\Phi$ of the form

$$
3 e_{\max } G\left(1-\left(\frac{x^{1}}{a_{1}}\right)^{2}\right)^{2} \delta\left(x^{2}\right) \delta\left(x^{3}\right) \Theta\left(a_{1}^{2}-x_{1}^{2}\right)
$$

where $\delta(x)$ and $\Theta(x)$ denote the Dirac delta distribution and the Heaviside step function, respectively. In cylindrical coordinates $\left(x^{2}=\rho \cos \varphi\right.$ and $\left.x^{3}=\rho \sin \varphi\right)$ the solution to Equation (24a) reads

$$
\begin{aligned}
\Phi= & \frac{e_{\max } G}{8 a_{1}^{4}}\left(\sqrt{\left(a_{1}+x^{1}\right)^{2}+\rho^{2}}\left(-9 a_{1} \rho^{2}+58 a_{1}^{2} x^{1}+26 a_{1}\left(x^{1}\right)^{2}-18 a_{1}^{3}+55 \rho^{2} x^{1}-50\left(x^{1}\right)^{3}\right)+\right. \\
& \sqrt{\left(a_{1}-x^{1}\right)^{2}+\rho^{2}}\left(-9 a_{1} \rho^{2}-58 a_{1}^{2} x^{1}+26 a_{1}\left(x^{1}\right)^{2}-18 a_{1}^{3}-55 \rho^{2} x^{1}+50\left(x^{1}\right)^{3}\right)+ \\
& \left.3\left(8 \rho^{2}\left(a_{1}^{2}-3\left(x^{1}\right)^{2}\right)+8\left(a_{1}^{2}-\left(x^{1}\right)^{2}\right)^{2}+3 \rho^{4}\right) \ln \left(\frac{\sqrt{\left(a_{1}-x^{1}\right)^{2}+\rho^{2}}+a_{1}-x^{1}}{\sqrt{\left(a_{1}+x^{1}\right)^{2}+\rho^{2}}-a_{1}-x^{1}}\right)\right) .
\end{aligned}
$$

Now consider the potentials $U_{\alpha}$. We remind the reader that $U_{3}$ vanishes for all $\bar{a}_{2}$. We prove that $U_{1}$ is also zero in the exterior in the limit by looking at its multipoles. The inhomogeneity in Equation (24b) is proportional to the Newtonian linear momentum density in the $x^{1}$-direction. Its integral over a slice $\mathcal{S}\left(\delta x^{1}, x_{0}^{1}\right)$ vanishes because of the antisymmetry of $v^{(0) 1}$. The integrals over the halves of the slice with $x^{2}>0$ and $x^{2}<0$ are given to leading order in $\bar{a}_{3}$ by

$$
P_{ \pm}^{1}=\mp 2 \frac{e_{\max }^{\frac{3}{4}}}{\pi G^{\frac{1}{4}}} \delta x^{1}\left(1-\left(\frac{x_{0}^{1}}{a_{1}}\right)^{2}+O\left(\frac{\delta x^{1}}{a_{1}}\right)\right)\left(-\ln \bar{a}_{3}\right)^{-\frac{1}{4}}
$$

which evidently tend to zero for $\bar{a} \rightarrow 0$. An arbitrary multipole moment for, e.g. the density $\mu v^{(0) 1}$, again to leading order, is then bounded by

$$
\int_{\mathcal{S}}\left|\mu v^{(0) 1}\left(x^{1}\right)^{i}\left(x^{2}\right)^{j}\left(x^{3}\right)^{k}\right| d^{3} x \leq\left(a_{1}\right)^{i}\left(a_{2}\right)^{j}\left(a_{3}\right)^{k} \int_{\mathcal{S}}\left|\mu v^{(0) 1}\right| d^{3} x=\left(a_{1}\right)^{i}\left(a_{2}\right)^{j}\left(a_{3}\right)^{k}\left(\left|P_{+}^{1}\right|+\left|P_{-}^{1}\right|\right),
$$

which all tend to zero by virtue of the preceding equation. This proves that $U_{1}$ vanishes for $\bar{a}_{2} \rightarrow 0$ in the exterior. This holds via corresponding arguments for $U_{2}$, too. Hence, the time-like Killing vector is hypersurface orthogonal and the spacetime is static in the limit.

It remains to analyze $\delta U$ and the PN surface. With the mass density given in Equation (C1) one can show that $U$ tends to zero in the exterior analogously to $U_{\alpha}$. It, thus, suffices to consider the solution to the Poisson equation for $U^{\prime}$ from Equation (21). The $1 \mathrm{PN}$ surface is still defined by the condition of vanishing pressure at the surface. A necessary condition for the inhomogeneity in Equation (21) to have a well-defined limit in a distributional sense is that the mass $\delta M$ contained in a slice $\mathcal{S}$ is well-defined to $1 \mathrm{PN}$ order:

$$
\delta M=\int_{x_{0}^{1}}^{x_{0}^{1}+\delta x^{1}} \int_{0}^{2 \pi} \int_{0}^{\rho_{s}} \mu \rho d \rho d \varphi d x^{1}=\frac{\mu}{2} \int_{x_{0}^{1}}^{x_{0}^{1}+\delta x^{1}} \int_{0}^{2 \pi} \rho_{s}^{2} d \rho d \varphi d x^{1}
$$


where $\rho_{s}$ denotes the $1 \mathrm{PN}$ surface (20) in cylindrical coordinates. Using our standard notation for PN terms, the $1 \mathrm{PN}$ order of the relevant term of the integrand is

$$
\left(\rho_{s}\left(x^{1}, \varphi\right)\right)^{2}=\left(\rho^{(0)}\left(x^{1}, \varphi\right)\right)^{2}+2 \rho^{(0)}\left(x^{1}, \varphi\right) \rho^{(2)}\left(x^{1}, \varphi\right) c^{-2} .
$$

The integral over the Newtonian contribution vanishes because of Equation (C1). The second term can be written out explicitly using Equation (25) and an expansion in terms of $\bar{a}_{3}$. The expansions of $w_{j}$ and $S_{i}$ will be denoted by

$$
\begin{aligned}
& w_{j}=w_{j 0}\left(\ln \bar{a}_{3}\right)+w_{j 2}\left(\ln \bar{a}_{3}\right) \bar{a}_{3}^{2}+O\left(\bar{a}_{3}^{3}\right), \quad j \in\{1,2\}, \\
& S_{i}=\left\{\begin{array}{l}
S_{i 0}\left(w_{j k}, \ln \bar{a}_{3}\right)+S_{i 2}\left(w_{j k}, \ln \bar{a}_{3}\right) \bar{a}_{3}^{2}+O\left(\bar{a}_{3}^{3}\right), \quad i \in\{1,2,3\} \\
S_{i 0}\left(w_{j k}, \ln \bar{a}_{3}\right) \bar{a}_{3}^{-2}+S_{i 2}\left(w_{j k}, \ln \bar{a}_{3}\right)+O\left(\bar{a}_{3}^{3}\right), \quad i \in\{4,5\} .
\end{array}\right.
\end{aligned}
$$

The expression (C7) diverges like $\bar{a}_{3}^{-2}$ for $\bar{a}_{3} \rightarrow 0$ in general. Hence, $\delta M$ becomes singular in this limit as well, see Equation (C6). These diverging terms vanish only if we have:

$$
S_{50}=4 S_{10}, \quad S_{50}=-\frac{4}{3} S_{30}
$$

However, the $S_{i 0}$ must also be consistent with Equation (33) to leading order, which provides the further conditions

$$
\begin{aligned}
0= & 6 S_{10}+12 S_{20}-10 S_{40}-5 S_{50}, \\
0= & 4 S_{40}+S_{50} \\
0= & S_{10}\left(\frac{25}{12}+\ln \frac{\bar{a}_{3}}{2}\right)+\frac{S_{20}}{6}-\frac{S_{30}}{3}\left(\frac{31}{12}+\ln \frac{\bar{a}_{3}}{2}\right)-\frac{S_{40}}{36}-\frac{S_{50}}{2}\left(\frac{197}{72}+\ln \frac{\bar{a}_{3}}{2}\right)+\frac{w_{10}-w_{20}}{6} \sqrt{-\frac{3}{2}-\ln \frac{\bar{a}_{3}}{2}}, \\
0= & 6 S_{30}-10 S_{40}-11 S_{50}, \\
0= & 4\left(S_{10}-S_{20}-\frac{1}{2}\right)\left(\frac{3}{2}+\ln \frac{\bar{a}_{3}}{2}\right)-3 S_{30}\left(\frac{109}{54}+\ln \frac{\bar{a}_{3}}{2}\right)-2 S_{40}\left(\frac{73}{48}+\ln \frac{\bar{a}_{3}}{2}\right)-\frac{5}{4} S_{50}\left(\frac{23}{8}+\ln \frac{\bar{a}_{3}}{2}\right)+ \\
& \left(w_{10}+w_{20}\right) \sqrt{-\frac{3}{2}-\ln \frac{\bar{a}_{3}}{2}} .
\end{aligned}
$$

Together with Equations (C10) these equations imply that

$$
S_{i 0}=0 \quad i \in\{1,2, \ldots, 5\}, \quad w_{10}=w_{20}=\sqrt{-\frac{3}{2}-\log \frac{\bar{a}_{3}}{2}} .
$$

Although these conditions are necessary they are not sufficient. The next to leading order in $\bar{a}_{3}$ in $\delta M$ diverges in general logarithmically. To choose the parameters $w_{i 2}$ so that these terms vanish, we solve first 
the system of equations originating from Equation (33), which reads to this order

$$
\begin{aligned}
0= & 6 S_{12}+12 S_{22}-10 S_{42}-5 S_{52}+16 w_{10}^{2} \\
0= & 4 S_{42}+S_{52}+4 w_{10}^{2} \\
0= & S_{12}\left(12 w_{10}^{2}-7\right)-2 S_{22}-S_{32}\left(\frac{13}{3}-4 w_{10}^{2}\right)+\frac{1}{3} S_{42}+S_{52}\left(\frac{89}{12}-6 w_{10}^{2}\right)-2 w_{10}\left(w_{12}-w_{22}\right)-16 w_{10}^{4}+ \\
& \frac{8}{3} w_{10}^{2}+5=0 \\
0= & 6 S_{32}-10 S_{42}-11 S_{52}+40 w_{10}^{2} \\
0= & 96 w_{10}^{2}\left(S_{12}-S_{22}\right)+S_{32}\left(\frac{112}{3}-72 w_{10}^{2}\right)+S_{42}\left(1-48 w_{10}^{2}\right)+S_{52}\left(\frac{165}{4}-30 w_{10}^{2}\right)+24 w_{10}\left(w_{12}+w_{22}\right)- \\
& 144 w_{10}^{4}-100 w_{10}^{2}+5 .
\end{aligned}
$$

This can easily be solved and the solution, which depends on $w_{i 2}$, can be inserted in $\delta M$. Now, the vanishing of the diverging terms requires the following behavior of the free parameters $w_{i 2}$ :

$$
\begin{aligned}
& w_{12}=-\frac{21}{2}\left(-\log \bar{a}_{3}\right)^{\frac{3}{2}}+C_{1}\left(-\log \bar{a}_{3}\right)^{\frac{1}{2}}+C_{2}+O\left(\left(-\log \bar{a}_{3}\right)^{-\frac{1}{2}}\right), \\
& w_{22}=C_{3}\left(-\log \bar{a}_{3}\right)^{\frac{3}{2}}+C_{4}\left(-\log \bar{a}_{3}\right)+C_{5}\left(-\log \bar{a}_{3}\right)^{\frac{1}{2}}+C_{6}+O\left(\left(-\log \bar{a}_{3}\right)^{-\frac{1}{2}}\right)
\end{aligned}
$$

with the free constants $C_{i}$.

The constants $C_{1}, C_{2}, C_{3}$ and $C_{4}$ in Equation (C14) govern the length of the rod $\Delta x_{1}$ in the limit $\bar{a}_{3} \rightarrow 0$, which still diverges in general. The choice

$$
C_{3}=\frac{1}{134}\left(-16715+9072 \log 2+C_{1}\right)
$$

ensures a finite length, which reads then

$$
\left(\Delta x_{1}\right)^{2}=a_{1}^{2}-\frac{a_{1}^{2}\left(e_{\max } G\right)^{\frac{1}{2}}}{15 c^{2}}\left(288 C_{2}-67 C_{4}\right) .
$$

With an argument like in Equation (C5), one can show that all multipole moments converge with the choices (C14) and (C15) and that $\delta U$ is well-defined; the inhomogeneity in Equation (24b) tends to a line mass density, which is a polynomial in $x^{1}$ to order 4 :

$$
\frac{3 G e_{\max }}{134 a_{1}^{4} c^{2}}\left(-455+252 \log 2+16 C_{1}\right)\left(a_{1}^{4}-6 a_{1}^{2} x_{1}^{2}+5 x_{1}^{4}\right) \delta\left(x^{2}\right) \delta\left(x^{3}\right) \Theta\left(a_{1}^{2}-x_{1}^{2}\right) .
$$

This is already of order $c^{-2}$. Hence, it is sufficient to take the Newtonian length of the rod into account. The function $\delta U$ can easily be calculated for the line density (C17) and is given by

$$
\begin{aligned}
\delta U=- & \frac{e_{\max } G}{1072 a_{1}^{4}}\left(-455+252 \log 2+16 C_{1}\right)\left[N_{+}+N_{-}+\log \left(\frac{-a_{1}-x_{1}+\left(\left(a_{1}+x_{1}\right)^{2}+\rho^{2}\right)^{\frac{1}{2}}}{a_{1}-x_{1}+\left(\left(a_{1}-x_{1}\right)^{2}+\rho^{2}\right)^{\frac{1}{2}}}\right) \times\right. \\
& \left.\left(24 a_{1}^{4}-144 a_{1}^{2} x_{1}^{2}+120 x_{1}^{4}+72 a_{1}^{2} \rho^{2}-360 x_{1}^{2} \rho^{2}+45 \rho^{4}\right)\right], \\
N_{ \pm}= & \left(\left(a_{1} \pm x_{1}\right)^{2}+\rho^{2}\right)^{\frac{1}{2}}\left(42 a_{1}^{3} \mp 146 a_{1}^{2} x_{1}-130 a_{1} x_{1}^{2} \pm 250 x_{1}^{3}+45 a_{1} \rho^{2} \mp 275 x_{1} \rho^{2}\right) .
\end{aligned}
$$

With $\Phi, \delta U, U_{\alpha}$, and $U$ all metric functions are determined. The metric describes an axially symmetric and static vacuum and, therefore, belongs to the Weyl class. The matter region degenerates to a rod along 
the $x_{1}$-axis and is described by a singularity. The singular behavior can be read off the Kretschmann scalar and is given to leading order in $\rho$ by

$$
R^{a b c d} R_{a b c d} \sim \frac{1}{\rho^{4}\left(\log \frac{\rho}{a_{1}}\right)^{2}}
$$

The class of Weyl metrics includes such prominent members as the Lanczos metric and the Curzon-Chazy metric (for an overview of these two metrics, see, e.g. Griffiths \& Podolsky (2009)). These metrics result also in some special limits of the 1 PN Dedekind ellipsoids. First the limit $a_{3} \rightarrow 0$ is carried out as described above and afterward the following limits are taken: $a_{1} \rightarrow \infty$ in the case of the Lanczos metric and $a_{1} \rightarrow 0$ in case of the Curzon-Chazy particle. In the former case, the line densities (C2) and (C17) become

$$
3 e_{\max } G \delta\left(x^{2}\right) \delta\left(x^{3}\right), \quad \frac{3 G e_{\max }}{134}\left(-455+252 \log 2+16 C_{1}\right) \delta\left(x^{2}\right) \delta\left(x^{3}\right),
$$

which are constant along the entire $x^{1}$-axis. The resulting spacetime is cylindrically symmetric. The leading order in $c^{-1}$ of the mass parameter is vanishing, such that it can be interpreted as an effective gravitational mass per unit length, cf. Israel (1977); Griffiths \& Podolsky (2009).

In the case $a_{1} \rightarrow 0$, the density (C17) vanishes and the density (C2) tends to the point density $E \delta\left(x^{1}\right) \delta\left(x^{2}\right) \delta\left(x^{3}\right)$ with $E$ is the total Newtonian energy concentrated in this point. This coincides with a $1 \mathrm{PN}$ approximation to the Curzon-Chazy solution with a parameter $m$ with a vanishing leading order in $c^{-1}$.

\section{The plots of the solutions}

As some analytical expressions are lengthy they were at some places suppressed in the article. In Figure 2 , the main parameters describing the solution are plotted. We use for all parameters, say, $t_{1}$ the usual splitting $t_{1}=t_{1,0}+t_{1,1} \hat{w}_{1}+t_{1,2} w_{2}$ with the parameterization from Section 3.4.2 Subsequently, the coefficients $t_{1, i}$ are depicted. We use solid lines for $i=0$, dashed lines for $i=1$, and dot-dashed lines for $i=2$. This parameterization has the advantage that the singularity is already removed. The results reflecting the parameterization in Paper II are recovered if we set $\hat{w}_{1}=-\frac{0.354937}{\lambda}$ and $w_{2}=0$, which implies $w_{1}=0$, cf. Equation (37). In Figure 3, three qualitatively different cases, cf. Section 3.4.3, of the surfaces of the 1 PN Dedekind ellipsoids are shown.

\footnotetext{
${ }^{7}$ We use here the same notation as in Griffiths \& Podolskv (2009).
} 

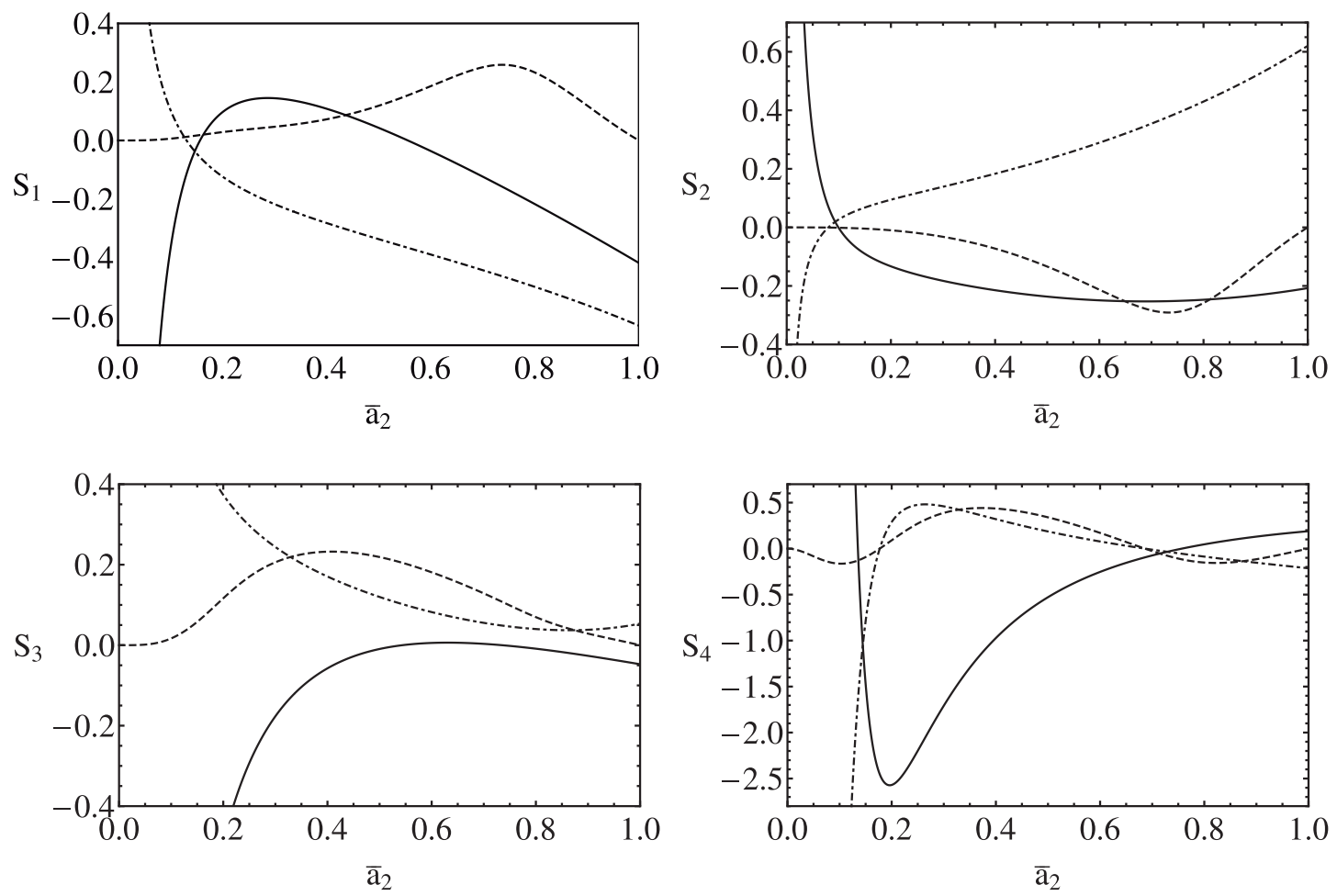

Fig. 2.- The parameters $S_{i}, t_{i}, r_{i}$ and $q_{i}$ describing the $1 \mathrm{PN}$ corrections of the surface and the velocity field, cf. Equation (25). 

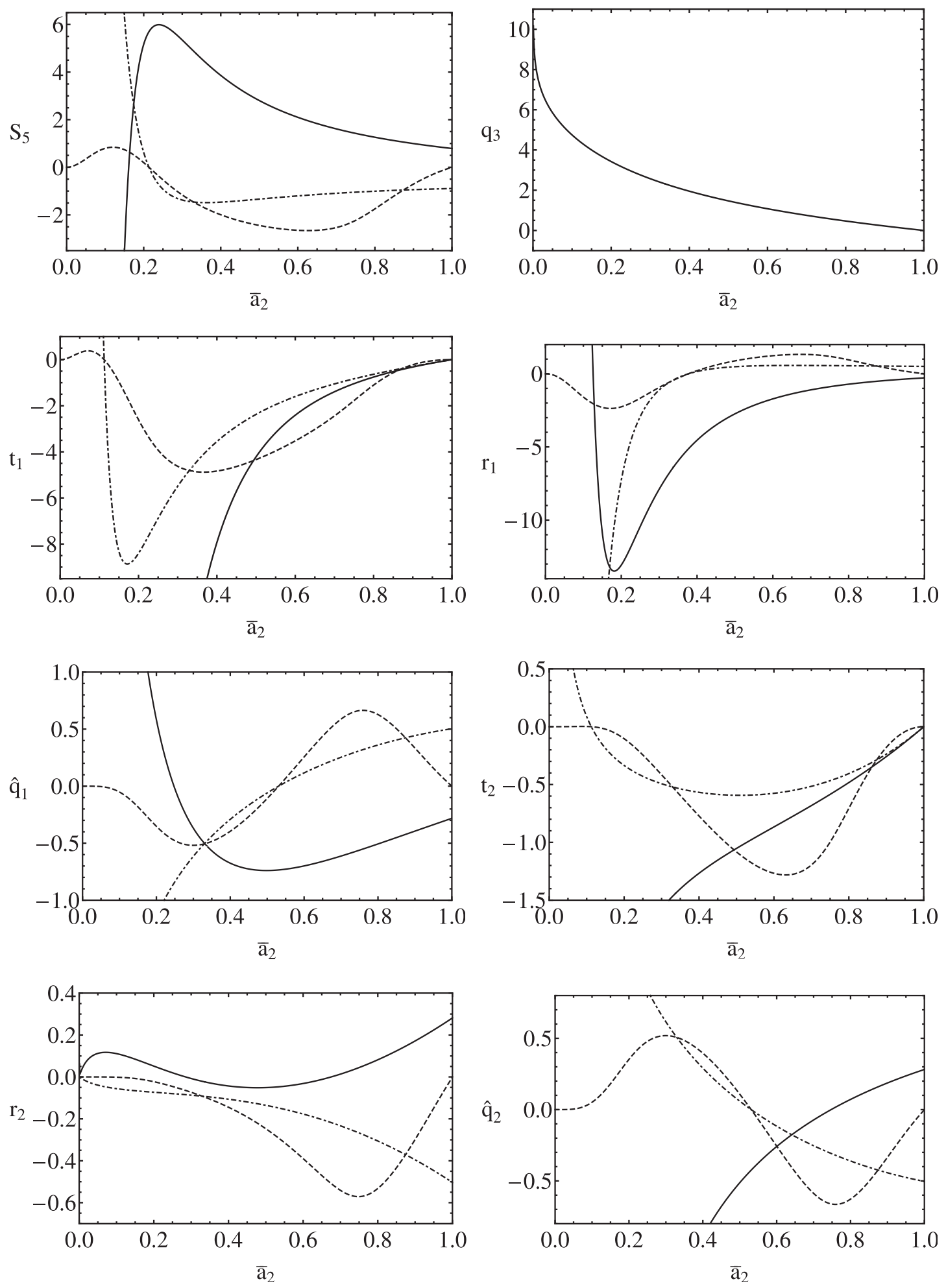

Fig. 2.- continued 


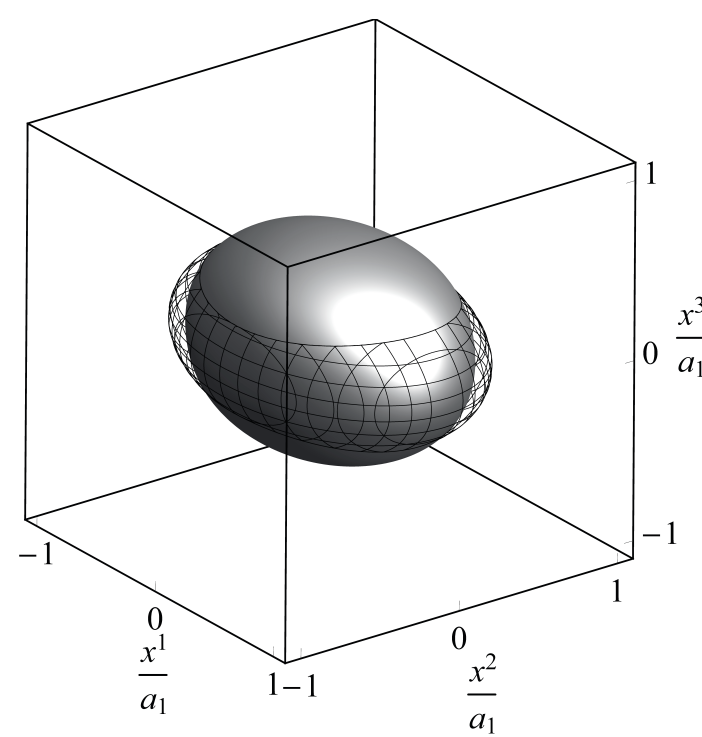

(a) $\hat{w}_{1}=0.164537, w_{2}=0.4\left(w_{1}=0.4\right)$

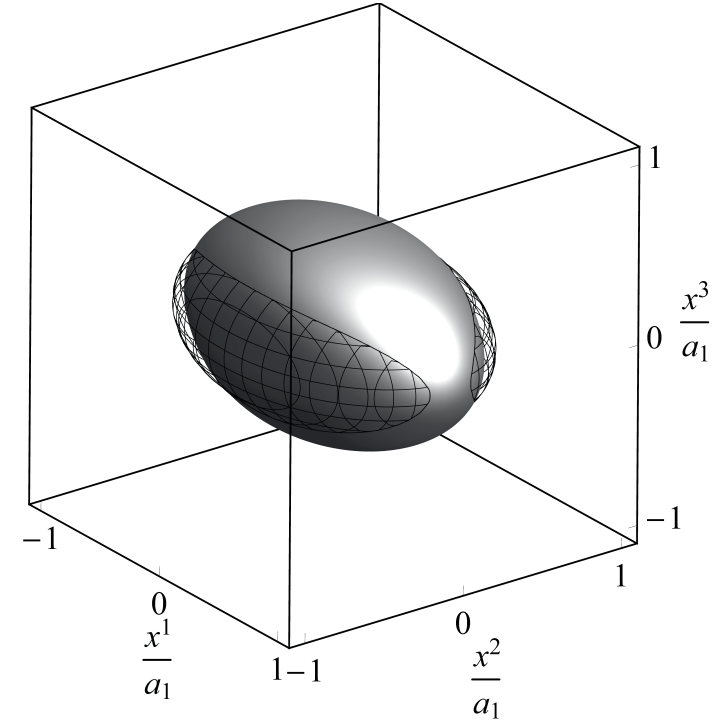

(b) $\hat{w}_{1}=0.468120, w_{2}=0\left(w_{1}=0\right)$, cf. Chandrasekhar \& Elbert (1974)

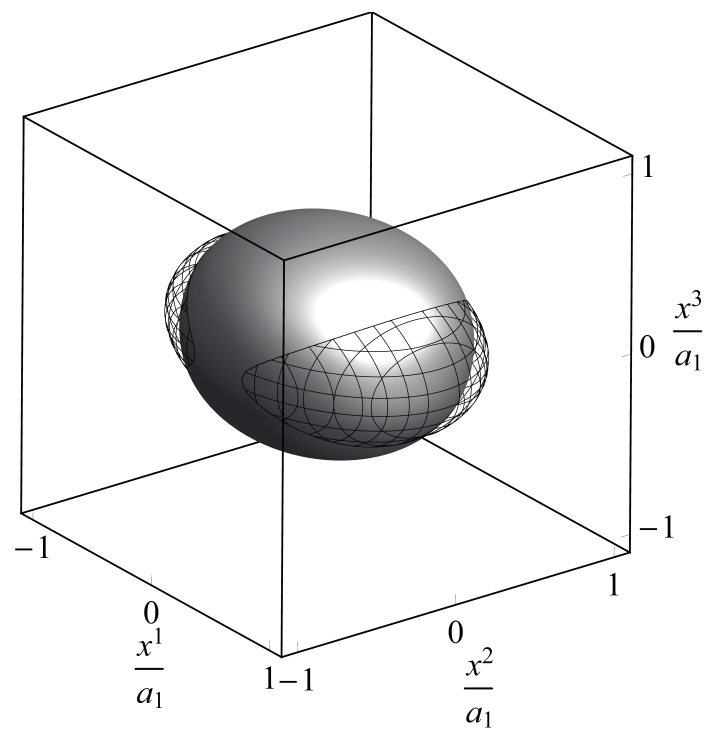

(c) $\hat{w}_{1}=-0.007157, w_{2}=0.8\left(w_{1}=0.7\right)$

Fig. 3.- The $1 \mathrm{PN}$ surfaces (solid, gray) compared to the Newtonian surface (wireframe, black) for parameters $\bar{a}_{2}=0.7$ and $\frac{G \mu}{c^{2}} a_{1}^{2}=0.15$ and different choices of the $\hat{w}_{1}$ and $w_{2}$. 